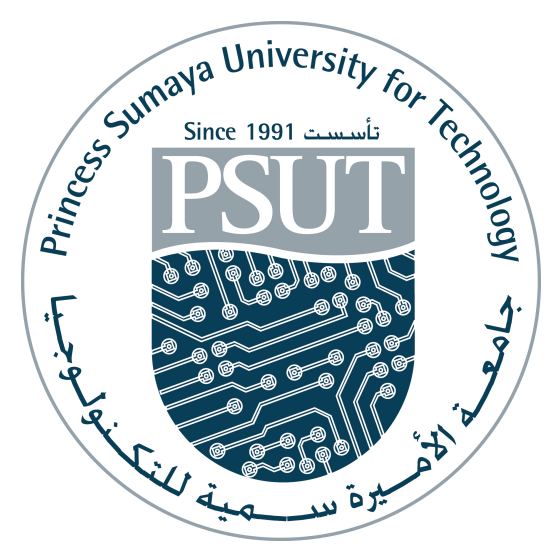

Design of a Modular Multiplier for Public-Key

Cryptography Applications Using Residue

Number System and Signed-Digit Representation

\author{
By \\ Mohammad Hizzani
}

Supervisor

Prof. Ahmad Hiasat

Thesis Submitted in Partial Fulfillment of the Requirements for the Degree of Master of Science in Electrical Engineering

Princess Sumaya University for Technology

King Abdullah I school of Graduate Studies and Scientific Research

May, 2019 


\section{Princess Sumaya University for Technology King Abdullah I School of Graduate Studies and Scientific Research Authorization Form}

I, Mohammad Hizzani, authorize Princess Sumaya University for Technology to supply copies of my Thesis/Dissertation to library or establishments or individuals on request, according to the regulations of Princess Sumaya University for Technology.

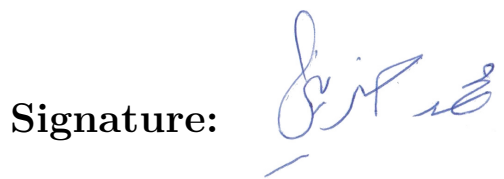

Date: Tuesday $28^{\text {th }}$ May, 2019 


\section{Examination Committee Decision}

This Thesis (Design of a Modular Multiplier for Public-Key Cryptography Applications Using Residue Number System and Signed-Digit Representation) was Successfully Defended and Approved on 28/5/2019.

Examination Committee

$\underline{\text { Signature }}$

Prof. Ahmad A. Hiasat, (Supervisor), Chairman. Professor of Computer Engineering

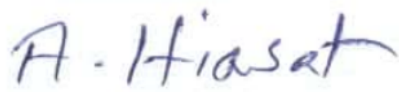

Prof. Ja'afer M. Al-Saraireh, Member

Professor of Computer Science

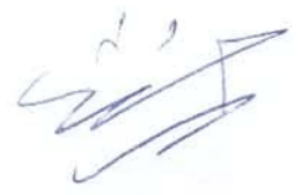

Dr. Osama D. AlKhaleel, Member

Associate Professor of Computer Engineering

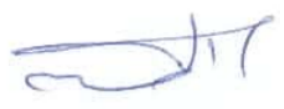

Dr. Lo'ai A. Tawalbeh, Member

Associate Professor of Computer Engineering

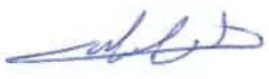




\title{
Design of a Modular Multiplier for Public-Key Cryptography Applications Using Residue Number System and Signed-Digit Representation
}

\author{
By \\ Mohammad Hizzani
}

Supervisor

Prof. Ahmad Hiasat

\section{Abstract}

Public-Key Cryptosystems are prone to wide range of cryptanalyses due to its property of having key pairs one of them is public. Therefore, the recommended length of these keys is extremely large (e.g. in Rivest-Shamir-Adleman (RSA) and Diffie-Hellman (D-H) the key is at least 2048 bits long) and this leads the computation of such cryptosystems to be slower than the secret-key cryptosystems (i.e. Advanced Encryption Standard (AES) and AES-family). Since, the key operation in such systems is the modular multiplication; in this research a novel design for the modular multiplication based on the Montgomery Multiplication, the Residue Number Systems for moduli of any form, and the Signed-Digit Representation is proposed. The proposed design outperforms the current designs in the literature in terms of delay with at least $28 \%$ faster for the key of 2048 bits long. Up to our knowledge, this design is the first design that

utilizes Signed-Digit Representation with the Residue Number System for moduli of any form. 


\section{Dedication}

To my beloved mother who believes in me like no one other

To my father who died before seeing this moment

To my brilliant brothers whom I count on and for sure they count on me

To my brother-friend who never let me down and always cheers me up

To all my teachers who built me piece by piece

I dedicate this work

Mohammad Hizzani 


\section{Acknowledgment}

Praises to Allah almighty, he blessed me with power and determination to do this work.

I would like to express my sincere gratitude and deep thankfulness to my supervisor, Prof. Ahmad Hiasat, for his continuous and his priceless support and guidance. He motivated me and was a role model for me to do this work. Without him it was impossible to do this.

Not to forget Ms. Ghadeer Abu Lail, Secretary of Electrical Engineering Department, for her countless efforts and support for me and for all students in our department during my study at Princess Sumaya University for Technolog (PSUT).

I would like to send a special thank to Prof. Christof Paar for his wonderful videos on YouTube explaining cryptography.

Special thanks to all my friends and colleagues at PSUT for all their help and support.

I would like to spotlight that this research was done under the generous sponsorship of HOPES organization. Thank you HOPES for your support and sponsorship.

Finally, I would like to thank the examiners, Prof. Jaafer Al-Saraireh, Dr. Osama AlKhaleel and Dr. Lo'Ai Tawalbeh for their valuable discussions and insightful comments, and for their good words about my work that charged me with positive energy to keep working at the same level of passion.

Mohammad Hizzani 


\section{List of Contents}

Authorization $\ldots \ldots \ldots \ldots \ldots \ldots$ ii

Examination Committee Decision $\ldots \ldots \ldots \ldots$ iii

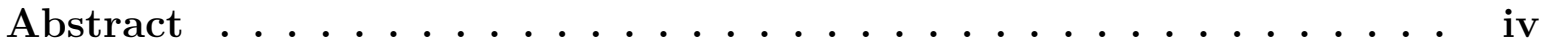

Dedication .....................

Acknowledgment $\ldots \ldots \ldots \ldots \ldots \ldots \ldots \ldots \ldots$ vi

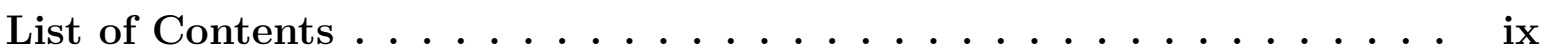

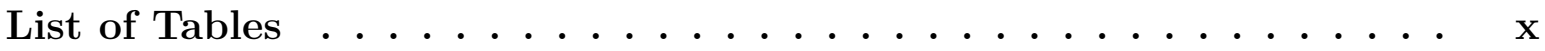

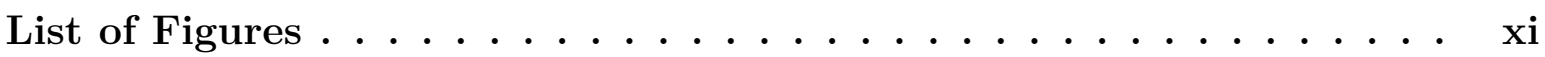

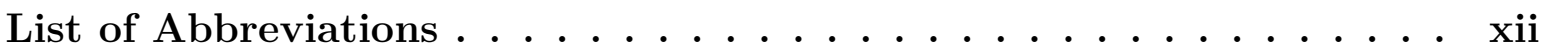

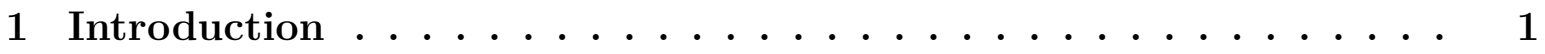

1.1 Application-Specific Arithmetic Operations . . . . . . . . . . . . . . 1

1.2 Arithmetic Operations in Cryptography . . . . . . . . . . . 2

1.2.1 Public-Key Cryptography . . . . . . . . . . . . . . 3

1.2.2 Modular Exponentiation . . . . . . . . . . . . . . 5

1.2.3 Modular Multiplication . . . . . . . . . . . . . 6

1.3 Signed-Digit Representation and Carry-Free Addition . . . . . . . . 7 
1.4 Residue Number System (RNS) . . . . . . . . . . . . . . . . . . 8

1.4.1 Forward and Reverse Conversion . . . . . . . . . . . 8

1.4.2 Operations Within RNS Channels . . . . . . . . . . . . 10

2 Literature Review . . . . . . . . . . . . . . . . . 12

2.1 Montgomery Multiplier . . . . . . . . . . . . . . . . . . . . . . . 12

2.2 Montgomery Multiplier Using RNS _ . . . . . . . . . . . . . . . 13

2.3 Previous Works . . . . . . . . . . . . . . . . . . . 14

2.3.1 Modular Adder . . . . . . . . . . . . . . . . . . . . . 15

2.3 .2 Modular Multiplier . . . . . . . . . . . . . . . . . . 15

2.3 .3 Base Extension . . . . . . . . . . . . . . . . . . . . . 16

3 Proposed Designs $\ldots \ldots \ldots \ldots \ldots \ldots \ldots \ldots$

3.1 Modular Adder Using SDR . . . . . . . . . . . . . . . . . 17

3.2 Modular Multiplier Using SDR . . . . . . . . . . . . . . . . . . 22

3.2 .1 Division-During-Multiplication $\ldots \ldots \ldots \ldots \ldots \ldots$

3.2 .2 Division-After-Multiplication $\ldots \ldots \ldots \ldots \ldots$

3.3 Base Extension Using SDR . . . . . . . . . . . . . . . . . . 26

3.3.1 Chinese Remainder Theorem (CRT) vs Mixed-Radix Conversion

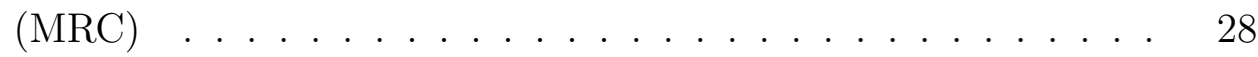

4 Complexity Analysis . . . . . . . . . . . . . . 32

4.1 Complexity Analysis . . . . . . . . . . . . . . . . . . 32

4.1.1 Complexity of SDR Modular Adder . . . . . . . . . . . . 33

4.1.2 Complexity of SDR Modular Multiplier _ . . . . . . . . 37

4.1 .3 Complexity of SDR Base Extension . . . . . . . . . . . . 42

4.2 Complexity Comparison . . . . . . . . . . . . . . . . 45

4.2.1 Complexity Comparison of Modular Addition . . . . . . . . 46

4.2.2 Complexity Comparison of Modular Multiplication . . . . . . 48 
4.2.3 Complexity Comparison of Base Extension . . . . . . . . . .

Recommendations and Future Work ............... 52

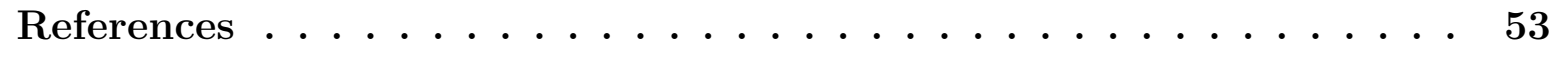

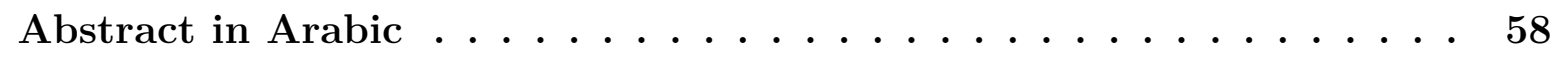




\section{List of Tables}

\begin{tabular}{|ll|c|}
\hline \multicolumn{1}{|c|}{ Table } & Page no. \\
\hline Table 1.1: & \multicolumn{1}{|c|}{ Repeated-Square-and-Multiply algorithm example } & 6 \\
\hline Table 3.1: $\begin{array}{l}\text { Signed-Digit Representaion (SDR) Addition Calcula- } \\
\text { tions }\end{array}$ & 20 \\
\hline Table 3.2: & SDR Modular Addition's Modular Reduction Step & 21 \\
\hline Table 3.3: & SDR Multiplier Recoding Rules & 25 \\
\hline Table 3.4: & SDR Recoded Multiplier Multiplication Rule & 28 \\
\hline Table 4.1: & SDR Encoding & 33 \\
\hline Table 4.2: & SDR Radix-4 Encoding & 38 \\
\hline Table 4.3: & $\begin{array}{l}\text { The values of the parameters used in this research design } \\
\text { example. }\end{array}$ & 50 \\
\hline Table 5.1: & Complexity Comparison Between the Proposed Design & 51 \\
\hline
\end{tabular}




\section{List of Figures}

\begin{tabular}{|c|c|}
\hline Figure & Page no. \\
\hline Figure 1.1: D-H Key Exchange Algorithm & 4 \\
\hline Figure 1.2: RSA Key Generating, Encryption, Decryption Algorithm & 5 \\
\hline Figure 1.3: SDR Addition Example & 8 \\
\hline Figure 3.1: SDR Modular Addition Examples & 20 \\
\hline Figure 3.2: Doubling-Accumulating Process For Parallel-Series Operation & 24 \\
\hline Figure 3.3: SDR Addition Tree Example & 27 \\
\hline Figure 3.4: SDR Addition Element & 28 \\
\hline Figure 3.5: SDR Base Extension & 31 \\
\hline Figure 4.1: SDR Modular Adder & 34 \\
\hline Figure 4.2: SDR One-Bit Partial SumCarry (PSC) & 35 \\
\hline Figure 4.3: $\quad$ SDR PSC TwoBitSum & 35 \\
\hline Figure 4.4: SDR PSCless & 36 \\
\hline Figure 4.5: SDR TwoBitSumless & 36 \\
\hline Figure 4.6: SDR recPSC & 39 \\
\hline Figure 4.7: SDR recTwoDigSum & 39 \\
\hline
\end{tabular}




\section{List of Abbreviations}

\begin{tabular}{|l|l|}
\hline Abbreviation & \\
\hline AES & Advanced Encryption Standard \\
\hline AKC & Asymmetric-Key Cryptography \\
\hline ASIC & Application Specific IC \\
\hline BR & Barrett Reduction \\
\hline CLA & Carry Look-Ahead Adder \\
\hline CPU & Central Processing Unit \\
\hline CRT & Chinese Remainder Theorem \\
\hline D-H & Diffie-Hellman \\
\hline DES & Data Encryption Standard \\
\hline ECC & Elliptic Curve Cryptography \\
\hline FPGA & Field Programmable Gate Array \\
\hline GPP & General-Purpose Processor \\
\hline GPU & Graphical Processing Units \\
\hline IBR & Improved Barrett Reduction \\
\hline IC & Integrated Circuit \\
\hline IoT & Internet of Things \\
\hline LSB & Least Significant Bit(s) \\
\hline MM & Montgomery Multiplier \\
\hline MR & Modular Reduction \\
\hline MRC & Mixed-Radix Conversion \\
\hline MSB & Most Significant Bit(s) \\
\hline PKC & Public-Key Cryptography \\
\hline PPA & Parallel Prefix Adder \\
\hline PSC & Partial SumCarry \\
\hline PSUT & Princess Sumaya University for Technolog \\
\hline PrKC & Private-Key Cryptography \\
\hline RNS & Residue Number System \\
\hline RNSMM & Residue Number System Montgomery Multiplication \\
\hline ROM & Read Only Memory \\
\hline RSA & Rivest-Shamir-Adleman \\
\hline SDR & Signed-Digit Representaion \\
\hline SKC & Secret-Key Cryptography \\
\hline SKC & Secret-Key Cryptography \\
\hline UGA & Unit Gate Area \\
\hline UGD & \\
\hline VLSI & Verye Scale Integrated Circuit \\
\hline
\end{tabular}




\section{Chapter 1 Introduction}

Security in computers is an essential element in everyday use of the digital world. Cryptography is the key element in security. However, cryptography involves lots of highly complicated and computationally intensive mathematical operations, that consume big portion of computations times that include cryptography in it.

Software applications of these cryptosystems are not so efficient, especially when dealing with numerous amount of digital communications and Big Data. Some of these fields where cryptography is a must are Internet of Things (Internet of Things (IoT)s), Digital Cryptocurrency, Smart Grids, Auto-Driving Cars, military and intelligence applications, and many others.

\subsection{Application-Specific Arithmetic Operations}

General-Purpose Processors (General-Purpose Processor (GPP)) are powerful since they are fully-customized and engineered to provide best performance. Despite their great performance in general applications, they are not efficient when it comes to the long-word arithmetic operations such as these used in cryptography. These GPPs operate on word length of 64-bits at most. The current recommendation for the key length for cryptosystems such as Rivest-Shamir-Adleman RSA, and Diffie-Hellman D-H, exceeds 2048 bits. Hence, these GPPs consume a lot of time for a single operation (i.e. single Modular Exponentiation), which makes the computations for such cryptosystems the bottle-neck in any application.

Application specific designs are dedicated to special applications. Despite the fact that these designs are implemented using slower technology, they outperform any GPP 
when running such special applications. For instance, the current technology used for implementing these application specific ICs is $28 \mathrm{~nm}$, whereas the technology used for implementing the GPPs is less than $14 \mathrm{~nm}^{1}$. In some cases, it reaches $7 \mathrm{~nm}^{2}$. Still, these dedicated hardwares are much faster and more convenient for running these special applications.

Examples of these application-specific designs are Graphical Processing Units (Graphical Processing Units (GPU)), digital camera's processors, some storage units have a dedicated circuit to perform Advanced Encryption Standard (AES) ${ }^{3}$ and so forth.

Application-specific circuits can be implemented as an Application Specific Integrated Circuit (Application Specific IC (ASIC)). ASICs are sometimes integrated with the Central Processing Units (Central Processing Unit (CPU))s on the same chip. In such cases, ASIC operates as a co-processor that is utilized to speed up the running of the targeted application. The other implementation approach is the Field Programmable Gate Array (Field Programmable Gate Array (FPGA)) that helps to avoid the threat of Hardware Trojans.

These designs (i.e. application-specific circuits) can be implemented as an Application Specific IC (ASIC). The ASICs are sometimes being added to Central Processing Units (CPU)s to do these operations or it would be added to some other devices. Other implementations can be on a Field Programmable Gate Array (FPGA) to avoid the threat of Hardware Torjans.

\subsection{Arithmetic Operations in Cryptography}

Cryptography intends to hide information from unauthorized persons, and return it back to the authorized persons. Therefore, cryptosystems are categorized into two main categories based on the secrecy and similarity of the keys. Secret keys are use

\footnotetext{
${ }^{1}$ Intel, 2019 and Intel, 2018

${ }^{2}$ King, 2018; Gorss, 2016; Dignan, 2015; Summers, 2018; Shankland, 2018

${ }^{3}$ (UV500 2.5"/m.2/mSATA Encrypted SSD - 120GB-1.92TB / Kingston, 2018)
} 
for data encryption and decryption. The first category is Secret-Key Cryptography (Secret-Key Cryptography (SKC)), which also called Symmetric-Key Cryptography (Symmetric-Key Cryptography (SKC)). As the name implies, it is obvious that in these cryptosystems the key is private and symmetric (i.e. the same key used to perform both encryption and decryption). The second category is Public-Key Cryptography (Public-Key Cryptography (PKC)) also known as Asymmetric-Key Cryptography (Asymmetric-Key Cryptography (AKC)), where cryptosystems in this category use different keys for encryption and decryption. The keys in PKC come in pairs, a public key is used for encryption and verification of the digital signature and a private key is used for decryption and signing a message.

Under the category of SKC, there are lots of well-known cryptosystems. An example of such cryptosystems is Data Encryption Standard Data Encryption Standard (DES) (Standard \& Others, 1977) which is considered obsolete since 1991. However, an improved version called Triple-DES (Karn, Metzger, \& Simpson, 1995) is still used especially in credit cards and E-passports. Other new more secure cryptosystems are Advanced Encryption System AES (Daemen \& Rijmen, 1999), Twofish (Schneier et al., 1998), Serpent (Anderson et al., 1999), RC6 (Rivest et al., 1998), and MARS (Burwick et al., 1998).

Some Public-key cryptosystems are Rivest-Shamir-Adleman RSA (Rivest, Shamir, \& Adleman, 1978), Diffie-Hellman D-H (Diffie, Diffie, \& Hellman, 1976), Elgamal (Elgamal, 1985), and Elliptic Curve Cryptography Elliptic Curve Cryptography (ECC) which was introduced by (Koblitz, 1987) and (Miller, 1986) independently.

\subsubsection{Public-Key Cryptography}

AKC solved the problem of key distribution over an insecure channel, where D-H (Diffie et al., 1976) as shown in Figure 1.1 introduced their solution based on the mathemat-

ical hardness of Discrete Mathematics. The key pairs in D-H consists of public key 


\begin{tabular}{|c|c|}
\hline Alice & Bob \\
\hline \multicolumn{2}{|c|}{ public shared parameters } \\
\hline \multicolumn{2}{|c|}{$p \& g$} \\
\hline$a=$ generate secter random key & $b=$ generate secret random key \\
\hline Calculating Public Key, & Calculating Public Key, \\
\hline$A=g^{a} \bmod P$ & $B=g^{b} \bmod P$ \\
\hline \multicolumn{2}{|c|}{ exchange PK over insecure channel } \\
\hline \multicolumn{2}{|c|}{$A \rightarrow$} \\
\hline \multicolumn{2}{|c|}{$\leftarrow B$} \\
\hline Calculating shared secret key, & Calculating shared secret key, \\
\hline$K_{A B}=B^{a} \bmod P=g^{a b} \bmod P$ & $K_{A B}=A^{b} \bmod P=g^{a b} \bmod P$ \\
\hline
\end{tabular}

Figure 1.1: D-H Key Exchange Algorithm

$(P, g \& A$ or $B)$ where $p \& g$ are shared parameters through the system such that " $p$ " is big prime number and " $g$ " is a primitive root (i.e. generator in the cyclic group $G_{p}$ ) and the secret key is $(a$ or $b)$.

RSA (Rivest et al., 1978) introduced a complete cryptosystem for encryption, decryption, key exchange and digital signature. The upper part of Figure 1.2 depicts the key generation algorithm and the lower part depicts the encryption and decryption algorithm in RSA. The key pair consists of a public key $(n, e)$, which is used to encrypt the plain text $m$. The private key $(d)$ is used to decrypt the cipher text. RSA is based on the mathematical difficulty of prime numbers factorizing. Euler's totient function $\phi(n)$, is defined and calculated as follows:

$$
\phi(n)=(p-1) \cdot(q-1)
$$

where $n$ is the result of multiplication of two big prime numbers (i.e. $n=p \cdot q$ ). The private key $d$ is the multiplicative inverse of the public key $e$ modulo the Euler's totient function $\phi(n)$ and is defined by:

$$
1 \equiv d \cdot e \bmod \phi(n)
$$




\begin{tabular}{c|c}
\hline \multicolumn{1}{c|}{ Alice } & Bob \\
\hline \hline \multicolumn{2}{c}{ Alice generates key pair for once: } \\
\hline $\begin{array}{c}p \& q \\
\text { Generate two secret prime numbers, } \\
\text { Calculating the public key, } \\
n=p \times q\end{array}$ \\
\hline Generating random integer, \\
\hline Calculating the secret key, \\
$d \equiv e^{-1} \bmod \phi(n)$
\end{tabular}

Alice publishes her public key,

$$
(n, e) \rightarrow
$$

Bob sends message to Alice following:

\begin{tabular}{c|c}
\hline \hline & $\begin{array}{c}\text { Calculating the cipher text for the message } m \\
c \equiv m^{e} \bmod n\end{array}$ \\
\hline
\end{tabular}

Bob sends the cipher text,

\begin{tabular}{c|c}
\multicolumn{2}{c}{$\leftarrow c$} \\
\hline Decryption the cipher text, \\
$m \equiv c^{d} \bmod n$ &
\end{tabular}$-$

Figure 1.2: RSA Key Generating, Encryption, Decryption Algorithm

\subsubsection{Modular Exponentiation}

It is obvious that modular exponentiation is the main pillar in these cryptosystems (i.e. D-H and RSA). However, computing the modular exponentiation in the conventional way (i.e. repeated multiplication such as $2^{5}=2 \cdot 2 \cdot 2 \cdot 2 \cdot 2$ ) is very complex. Therefore, other algorithms are used to reduce the complexity of the exponentiation from $\mathcal{O}\left(2^{w}\right)$ (i.e. exponential complexity) to $\mathcal{O}(w)$ (i.e. linear complexity) where $w:=$ is the word length of the exponent. 
Table 1.1: Repeated-Square-and-Multiply algorithm example.

\begin{tabular}{|c||c|c|c|c|c|c|c|c|c|c|}
\hline$i$ & 0 & 1 & 2 & 3 & 4 & 5 & 6 & 7 & 8 & 9 \\
\hline$k_{i}$ & 0 & 0 & 1 & 0 & 1 & 0 & 1 & 0 & 0 & 1 \\
\hline$A$ & 5 & 25 & 625 & 681 & 1011 & 369 & 421 & 779 & 947 & 925 \\
\hline$b$ & 1 & 1 & 625 & 625 & 67 & 67 & 1059 & 1059 & 1059 & 1013 \\
\hline
\end{tabular}

Repeated square-and-multiply algorithm (Menezes et al., 1997, p. 71) is the most popular algorithm that reduces the exponentiation complexity and it is represented in Algorithm 1.

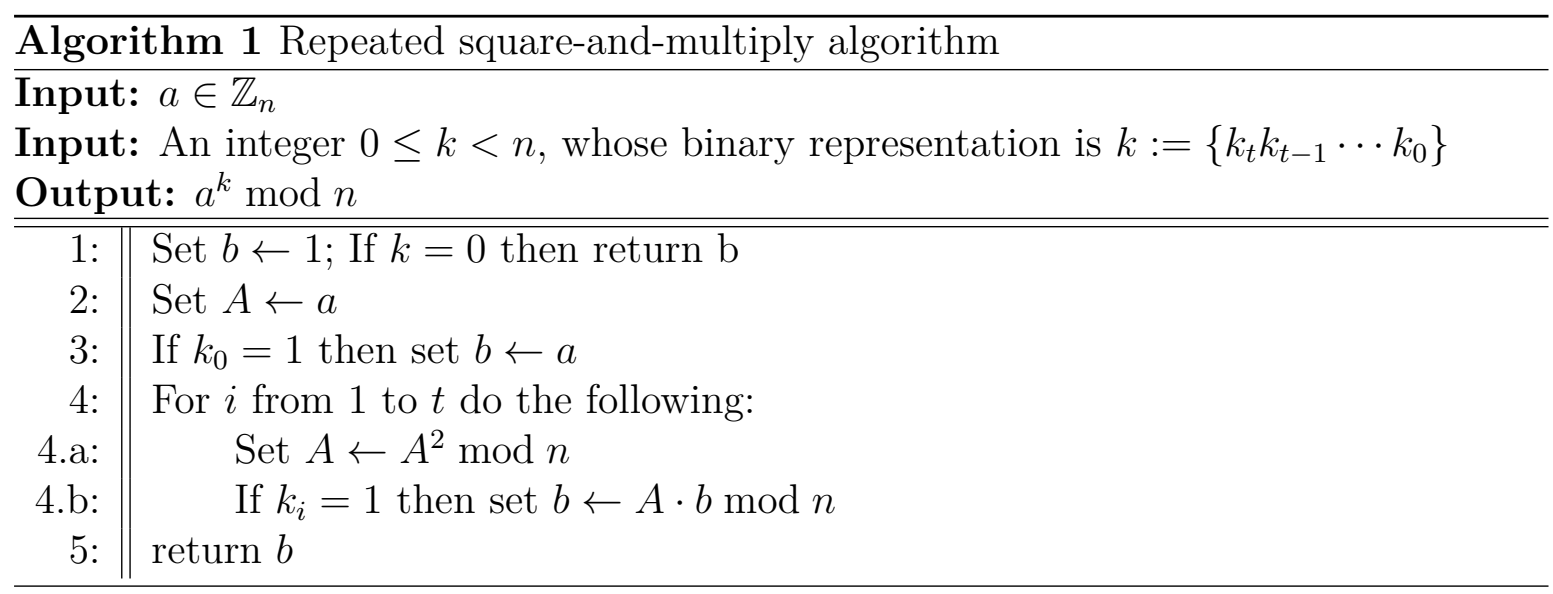

An example of applying repeated square-and-multiply algorithm that described in Algorithm 1 is shown in Table 1.1 to compute the following:

$$
c \equiv a^{k} \bmod n
$$

where $a=5, k=596$ and $n=1234$. Thus, $c=1013$.

\subsubsection{Modular Multiplication}

Algorithm 1 shows that the key operation in the modular exponentiation in cryptosystems such as D-H and RSA is the modular multiplication. Hence, many designs were introduced to improve this operation.

(Takagi \& Yajima, 1992) utilized the Signed-Digit Representation (Signed-Digit Representaion (SDR)) to design a modular multiplier with radix-4 re-coder. (Orup \& 
Kornerup, 1991) designed a higher radix re-coder.

Some designs introduced the idea of converting the calculation of modular reduction from the conventional trial division into multiplication. (Montgomery, 1985) introduced an algorithm that transformed the modular calculations into some multiplication and addition (see section 2.1). In addition, (Barrett, 1987) introduced Barrett Reduction algorithm (see section 3.2.2) and Dhem in (Dhem, 1994, 1998) developed a modified Barrett Reduction to reduce the error to zero.

\subsection{Signed-Digit Representation and Carry-Free Addition}

Signed-digit representation (SDR) (Avizienis, 1961) has great characteristics that can be utilized to improve the addition and subtraction operations. In SDR a number might have redundant representations with the same number of digits. This redundancy gives the ability to perform the addition in a carry-free manner. In binary number system, the SDR consists of three digits $\{\overline{1}, 0,1\}$.

The addition in SDR consists of two steps. The first step is to calculate the partial sum and partial carry as the following:

$$
\begin{gathered}
c_{i+1}= \begin{cases}1 & \text { if } x_{i}+y_{i}>1, \\
& \text { or } x_{i}+y_{i}=1 \text { and } x_{i-1}+y_{i-1}>\overline{1}, \\
0 & \text { if } x_{i}+y_{i}=0, \\
& \text { or } x_{i}+y_{i}=1 \text { and } x_{i-1}+y_{i-1} \leq \overline{1}, \\
& \text { or } x_{i}+y_{i}=\overline{1} \text { and } x_{i-1}+y_{i-1} \geq 1, \\
\overline{1} & \text { if } x_{i}+y_{i}<\overline{1},\end{cases} \\
u_{i}= \begin{cases}1 & \text { or } x_{i}+y_{i}=\overline{1} \text { and } x_{i-1}+y_{i-1}<1 \\
0 & \text { or } x_{i}+y_{i}=1 \text { and } x_{i-1}+y_{i-1} \leq \overline{1},\end{cases} \\
\overline{1} \quad \begin{array}{l}
\text { or } x_{i}+y_{i}>1 \text { or } x_{i}+y_{i}+y_{i-1}<1, \\
\text { if } x_{i}+y_{i}=1 \text { and } x_{i-1}+y_{i-1}>\overline{1},
\end{array}
\end{gathered}
$$




\begin{tabular}{rrrrrrr} 
& 1 & $\overline{1}$ & 0 & $\overline{1}$ & 1 & 7 \\
+ & 0 & $\overline{1}$ & 1 & 0 & 0 & -4 \\
\hline 0 & $\overline{1}$ & 0 & 0 & 1 & & $c_{i}$ \\
+ & 1 & 0 & 1 & $\overline{1}$ & $\overline{1}$ & $u_{i}$ \\
\hline 0 & 0 & 0 & 1 & 0 & $\overline{1}$ & 3
\end{tabular}

Figure 1.3: SDR Addition Example

The second step is a simple carry-free addition of the partial carry and the partial sum $\left(s_{i}=c_{i}+u_{i}\right)$. Figure 1.3 shows an example following the rules in equations 1.5 and 1.4.

\subsection{Residue Number System (RNS)}

Residue Number System Residue Number System (RNS) (Garner, 1959) is a nonweighted radix number system. Any integer in the set of $[0, M-1]$ can be represented using the following moduli set:

$$
M_{\text {set }}:=\left\{m_{1}, m_{2}, \cdots, m_{N}\right\}
$$

where $M$ is the dynamic range of the moduli set $M_{\text {set }}$ :

$$
M=\prod_{i=1}^{N} m_{i}
$$

and the elements of the moduli set are pairwise coprime (i.e. $\left.\operatorname{gcd}\left(m_{i}, m_{j}\right)=1, i \neq j\right)$.

\subsubsection{Forward and Reverse Conversion}

Any integer in $[0, M-1]$ has a unique representation in the moduli set $M_{\text {set }}:=$ $\left\{m_{1}, m_{2}, \cdots, m_{N}\right\}$ as:

$$
X=\left\{x_{1}, x_{2}, \cdots, x_{N}\right\}
$$

where;

$$
x_{i} \equiv X \bmod m_{i}=|X|_{m_{i}}
$$


An example where $M_{\text {set }}:=\{7,8,9\}$, so any integer in $[0, M-1]$ has a unique representation, where $M=\prod_{1}^{3} m_{i}=504$. Hence,

$$
\begin{aligned}
& 29=\left\{|29|_{7},|29|_{8},|29|_{9}\right\}_{M_{\text {set }}} \\
& 29=\{1, \quad 5, \quad 2\}_{M_{\text {set }}}
\end{aligned}
$$

The reverse conversion from RNS to conventional binary representation is preformed through two main algorithms, Chinese Remainder Theorem CRT and Mixed-Radix Conversion MRC.

CRT which is defined in Equation 1.12 needs some pre-onetime-computed numbers. First,

$$
M_{i}=\frac{M}{m_{i}}
$$

And it is being computed for each modulus in the moduli set. Second, $M_{i}^{-1}$ is computed, where,

$$
\begin{array}{r}
1 \equiv M_{i}^{-1} M_{i} \bmod m_{i} \\
X=\left.\left.\left|\prod_{1}^{N}\right| x_{i} M_{i}^{-1}\right|_{m_{i}} M_{i}\right|_{M}
\end{array}
$$

Hence, for the moduli set $\{7,8,9\}$ the $M_{i}$ and $M_{i}^{-1}$ arrays are:

$$
\begin{aligned}
M_{i} & =\{72,63,56\} \\
M_{i}^{-1} & =\{4,7,5\}
\end{aligned}
$$

Thus, converting the number $\{1,5,2\}$ into conventional binary representation using the CRT:

$$
\begin{gathered}
X=\left.|| 1 \cdot 4\right|_{7} \cdot 72+|5 \cdot 7|_{8} \cdot 63+\left.|2 \cdot 5|_{9} \cdot 56\right|_{504} \\
\mathrm{X}=29
\end{gathered}
$$

Performing the reverse conversion using the MRC (Huang, 1983) can be done as follows:

$$
X=x_{1}^{\prime}+u_{2} x_{2}^{\prime}+\cdots+u_{N} x_{N}^{\prime}
$$


where $u_{2}=m_{1}, u_{3}=m_{1} m_{2}, u_{4}=m_{1} m_{2} m_{3}$, and so forth. $x_{i}^{\prime}$ 's are computed as following:

$$
\begin{aligned}
x_{1}^{\prime} & =x_{1} \\
x_{2}^{\prime} & =\left.\left.\left|\left(x_{2}-x_{1}^{\prime}\right)\right| m_{1}^{-1}\right|_{m_{2}}\right|_{m_{2}} \\
x_{3}^{\prime} & =\left.\left.\left|\left(\left(x_{3}-x_{1}^{\prime}\right)\left|m_{1}^{-1}\right|_{m_{3}}-x_{2}^{\prime}\right)\right| m_{2}^{-1}\right|_{m_{3}}\right|_{m_{3}} \\
& \vdots \\
x_{N}^{\prime} & =\left.\left.\left|\left(\cdots\left(x_{N}-x_{1}^{\prime}\right)\left|m_{1}^{-1}\right|_{m_{n}}-\cdots-x_{N-1}^{\prime}\right)\right| m_{N-1}^{-1}\right|_{m_{N}}\right|_{m_{N}}
\end{aligned}
$$

Hence, converting the number $(X=\{1,5,2\})$ into conventional binary representation:

$$
X=x_{1}^{\prime}+u_{2} \cdot x_{2}^{\prime}+u_{3} \cdot x_{3}^{\prime}
$$

And $u_{i}{ }^{\prime}$ 's are:

$$
\begin{aligned}
& u_{2}=7 \\
& u_{3}=56
\end{aligned}
$$

The pre-computed multiplicative inverses are:

$$
\begin{aligned}
& \left|m_{1}^{-1}\right|_{m_{2}}=\left|7^{-1}\right|_{8}=7 \\
& \left|m_{1}^{-1}\right|_{m_{3}}=\left|7^{-1}\right|_{9}=4 \\
& \left|m_{2}^{-1}\right|_{m_{3}}=\left|8^{-1}\right|_{9}=8
\end{aligned}
$$

And $x_{i}^{\prime}$ s are:

$$
\begin{aligned}
& x_{1}^{\prime}=x_{1}=1 \\
& x_{2}^{\prime}=|(5-1) \cdot 7|_{8}=4 \\
& x_{3}^{\prime}=|((2-1) \cdot 4-4) \cdot 8|_{9}=0
\end{aligned}
$$

Eventually,

$$
X=1+7 \cdot 4+56 \cdot 0=29
$$

\subsubsection{Operations Within RNS Channels}

The most advantage of RNS that the operation (addition, subtraction and multiplication) can be performed without any carry across its channels. This is considered as a treasure especially in the case of public-key cryptography, since operation in such 
systems (RSA, D-H and Elgamal) are highly expensive in terms of delay due to the length of inputs (i.e. operands). The current recommendation for these cryptosystems for the bit-length is to be more than 2048 bits.

Arithmetic operations in RNS are as follows:

$$
X * Y=\left\{\left|x_{1} * y_{1}\right|_{m_{1}},\left|x_{2} * y_{2}\right|_{m_{2}}, \cdots,\left|x_{N} * y_{N}\right|_{m_{n}}\right\}
$$

where:

$$
* \equiv \times,+ \text { or }-
$$

Thus, the complexity of arithmetic operation got reduced significantly. For instance, when performing $(X \times Y)$ and they are assumed to be 2000-bit length. The complexity of such an operation using the conventional binary number system is much more than performing the same operation using 100-RNS-moduli set that each modulus length is 20 bits.

Although, utilizing RNS needs binary-to-residue conversion at the beginning and residue-to-binary conversion at the end, it is still much faster than conventional binary operations especially when performing modular exponentiation. When performing modular exponentiation with length of more than 2000 bit, more than 2000 modular multiplications are needed (see Section 1.2.2). However, forward and reverse conversion (to and from the RNS) are performed at the beginning and at the end of the whole modular exponentiation. 


\section{Chapter 2 \\ Literature Review}

\subsection{Montgomery Multiplier}

Montgomery Multiplier (Montgomery Multiplier (MM)) (Montgomery, 1985) is the most efficient algorithm to implement the modular multiplication using Residue Number System. Hence, it has been chosen as a subject of implementation in many designs (Antão, Bajard, \& Sousa, 2012), (Bajard, Kaihara, \& Plantard, 2009), (Asif \& Kong, 2017; Asif, Hossain, Kong, \& Abdul, 2018; Asif, Andersson, Rodrigues, \& Kong, 2018), (Bajard, Eynard, \& Gandino, 2013), (Antão \& Sousa, 2014),(Yang, Hu, \& Qin, 2015), (Yao, Fan, Cheung, \& Verbauwhede, 2014) and many more.

The algorithm suggests that in order to do any modular calculations each number should be transformed into the Montgomery Number System, which is simply the operands multiplied by the Montgomery Number modulo the modulus (i.e. the number to do modular reduction to) as:

$$
\begin{aligned}
& A^{\prime}=(A \times R) \bmod P \\
& B^{\prime}=(B \times R) \bmod P
\end{aligned}
$$

where $A \& B$ are inputs to the MM, $R$ is the Montgomery Number and $P$ is the modulus. Thus, the addition and subtraction in the Montgomery Number System need no further computation, as follows:

$$
\begin{aligned}
C & =A+B \bmod P \\
C^{\prime} & =C \times R \bmod P
\end{aligned}
$$


Addition is performed on numbers in the Montgomery Number System as follows:

$$
\begin{aligned}
A^{\prime}+B^{\prime} \bmod P & =(A+B) \times R \bmod P \\
& =C^{\prime}
\end{aligned}
$$

On the other hand, modular multiplication needs extra computation to keep the output in the Montgomery number system. These calculations are called Montgomery Reduction (Modular Reduction (MR)). As in Equation 2.2, a reduction algorithm is needed to reduce the $R^{2}$ into $R$ without changing the value of the modular multiplication. This process is being performed as in Algorithm 2 (Montgomery, 1985).

$$
A^{\prime} B^{\prime}=A B R^{2} \bmod P
$$

while

$$
\begin{aligned}
Z^{\prime} & =A B R \bmod P \\
& =Z R \bmod P
\end{aligned}
$$

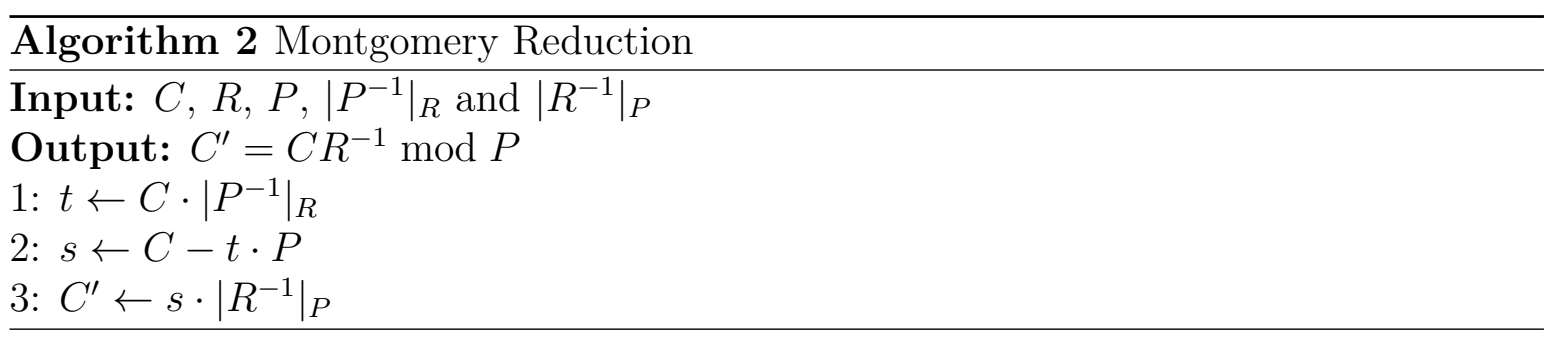

\subsection{Montgomery Multiplier Using RNS}

In the RNS, the Montgomery Number is the dynamic range of the moduli set $M_{\text {set }}:=$ $\left\{m_{1}, m_{2}, \cdots, m_{N}\right\} ; M=\prod_{i=1}^{N} m_{i}$. The problem in this case is that when applying the Montgomery Reduction, the output of step 2 in Algorithm 2 produces a zero. Thus, an auxiliary moduli set $\widetilde{M}_{\text {set }}:=\left\{\widetilde{m}_{1}, \widetilde{m}_{2}, \cdots, \widetilde{m}_{N}\right\}$ is needed, to transform the result of step 1. The procedure of transforming a number from one RNS moduli set into another RNS moduli set is called the Base Extension. 
In order to be able to perform the base extension - the auxiliary moduli set $\widetilde{M}_{\text {set }}$ must be relatively prime with $M_{\text {set }}$ (i.e. $\operatorname{gcd}(M, \widetilde{M})=1$ ). Algorithm 3 shows all steps of MM in RNS. Thus, RNS Montgomery Multiplication (Residue Number System Montgomery Multiplication (RNSMM)) has three main operations, modular multiplication within the channels of the moduli set, modular addition/ subtraction and base extension.

\begin{tabular}{|c|c|c|}
\hline \multicolumn{3}{|c|}{ Algorithm 3 RNS Montgomery Multiplication } \\
\hline \multicolumn{3}{|c|}{$\begin{array}{l}\text { Input: } M_{\text {set }}:=\left\{m_{1}, m_{2}, \cdots, m_{N}\right\}, \widetilde{M}_{\text {set }}:=\left\{\widetilde{m}_{1}, \widetilde{m}_{2}, \cdots, \widetilde{m}_{N}\right\} \\
\text { Input: } A^{\prime}, B^{\prime}, P \text { and }\left|P^{-1}\right|_{M} \text { all in both } M_{\text {set }} \text { and } \widetilde{M}_{\text {set }} \\
\text { Input: }\left|M^{-1}\right|_{P} \text { in } \widetilde{M}_{\text {set }} \\
\text { Output: } A^{\prime} B^{\prime} M^{-1} \bmod P\end{array}$} \\
\hline \multicolumn{2}{|c|}{ Base $M_{\text {set }}$ operations } & Base $\widetilde{M}_{\text {set }}$ operations \\
\hline 1: & $C \leftarrow A^{\prime} B^{\prime}$ & $C \leftarrow A^{\prime} B^{\prime}$ \\
\hline 2: & $t \leftarrow C\left|P^{-1}\right|_{M}$ & $\longrightarrow$ \\
\hline 3: & \multicolumn{2}{|c|}{ Base extension $\triangleright|t|_{\widetilde{M}} \Longleftarrow|t|_{M}$} \\
\hline 4: & - & $s \leftarrow C-t \cdot P$ \\
\hline 5: & & $C^{\prime} \leftarrow s \cdot\left|M^{-1}\right|_{P}$ \\
\hline $6:$ & \multicolumn{2}{|c|}{ Base extension $\triangleright\left|C^{\prime}\right|_{M} \Longleftarrow\left|C^{\prime}\right|_{\widetilde{M}}$} \\
\hline
\end{tabular}

\subsection{Previous Works}

As mention in the previous section, the RNSMM is divided into three main components in order to facilitate the study of each component. As per section 2.1, the main three components are Modular Adder, Modular Multiplier and Base Extension. In the following subsections the previous works on each component are presented. 


\subsubsection{Modular Adder}

Modular Adder represents an addition and a modular reduction. Since the inputs are less than the modulus (i.e. $0 \leq A \& B<m$ ), teh size of the result of the adition $(S=A+B)$ is 1-bit larger than the size of the modulus $m$. For example if $m$ is n-bit integer, then $S$ is $(\mathrm{n}+1)$-bit integer. Thus, the modular reduction can be a comparison with $m$ and an addition with $-m$ (i.e. if $S>m$ then $C=S-m$ ).

Implementing this design in the conventional binary operations can be achieved using any binary addition algorithm such as (Sklansky, 1960), (Kogge \& Stone, 1973) or (Brent \& Kung, 1982). All of these algorithms (also known as Parallel-Prefix Adders (Parallel Prefix Adder (PPA))) have a logarithmic complexity $\mathcal{O}\left(\log _{2}(n)\right)$. On the other hand, the comparison that follows the addition and the final addition (i.e. $-m$ ) also have a logarithmic complexity. The complexity of modular adder in the conventional binary representation is:

$$
\text { Delay }=3 \times(\text { delay of PPA })
$$

This research will utilize the advantage of the SDR to perform the modular addition with fixed complexity that is faster than any known modular addition designs in the literature. The reference design of this research is the modular adder designed by Takagi in (Takagi, 1992) and discussed in details later in section 3.1.

\subsubsection{Modular Multiplier}

Modular multiplier is divided into two main categories, one is the modular multiplier for long word operands (i.e. the operand are longer than 2000 bit such as in RSA and D-H) which is the target of this research as whole. The second is the RNS in-channel modular multiplication for short operands (i.e. shorter than 32 bits) which is a key operation in the RNSMM. Hence, many researches were focused on the development of this operation. 
(Asif \& Kong, 2017; Asif, Andersson, et al., 2018) exploited the modified Barrett reduction to design the modular multiplier for RNS in-channel operation to operate as a Montgomery modular multiplier.

\subsubsection{Base Extension}

Since base extension is a binary-to-residue conversion embedded in a residue-to-binary conversion (i.e. converting a number from a moduli set to another moduli set) there are two algorithms to perform the residue-to-binary conversion as mentioned in section 1.4.1.

(Schinianakis \& Stouraitis, 2011) exploited the MRC to design the base extension for RNSMM. The use of MRC over CRT is efficient in terms of no need to compute the

correction factor in the CRT. However, as per section 1.4.1, the calculations of $x_{i}^{\prime}$ s have a sequential form unlike the CRT.

Most of the researches in the literature focused on the exploitation of CRT. Since the CRT needs to compute the correction factor, (Shenoy \& Kumaresan, 1989) introduced the use of a redundant modulus to compute the correction factor. Since there are two base extensions in the single modular multiplier (Bajard, Didier, \& Kornerup, 2001) suggested to reserve and postpone the addition of the correction term of the first base extension to the second base extension. (Kawamura, Koike, Sano, \& Shimbo, 2000) suggested another approximation approach for the calculation of the correction factor.

Most of the work in the literature derived their designs from the above concepts introduced. For instance, (Antão \& Sousa, 2014) is a design on a GPU based on (Kawamura et al., 2000), (Asif \& Kong, 2017) is based on improved Barrett reduction (Dhem, 1994, 1998) for modular multiplier and on (Kawamura et al., 2000) for base extension. 


\section{Chapter 3 Proposed Designs}

In this chapter the proposed design is being introduced in details. This design is based on RNSMM algorithm that was introduced in section 2.2. The proposed design is divided into three main operations. The first operation is the Modular Addition as part of step 4 in algorithm 3, in addition to be a key element in the other two operations. The second operation is the Modular Multiplication as one of the main elements in the in-channel operations of RNSMM. The third operation is Base Extension which is the bottle neck -in terms of delay- of RNSMM.

The proposed design utilizes both number systems RNS and Signed-Digit Representation (SDR). The idea behind exploiting both systems is that each number system has an advantage for specific arithmetic operations. For instance, for long-word numbers, RNS creates parallel channels that can operate in parallel, which reduces significantly the delay of exponent-complexity operations such as binary multiplication and modular multiplication. Moreover, as in section 1.3, SDR performs addition and subtraction in a carry-free manner.

This research is being developed for any general moduli set. There is no condition on the form of the moduli set (i.e. some designs were developed for a specific form of moduli set such as $\left\{2^{n}-1,2^{n}, 2^{n}+1\right\}$ (Wei, 2009) and (Timarchi, Saremi, Fazlali, \& Gaydadjiev, 2013)).

\subsection{Modular Adder Using SDR}

Modular Addition is simply an addition followed by a modular reduction. Thus, the addition can be performed using any of the well-known adders such as Carry Look- 
Ahead Adder (Carry Look-Ahead Adder (CLA)) (i.e. Parallel Prefix Adder (PPA)) (Sklansky, 1960; Kogge \& Stone, 1973; Brent \& Kung, 1982). The modular reduction can be performed by simply comparing the number with the modulus and subtract the modulus if the result is bigger. Utilizing the carry-free addition property of SDR, (Takagi, 1992) built a modular adder that keeps the result truncated to $(n+1)$-bit length, unlike the conventional SDR addition (Avizienis, 1961), which has an output longer than inputs by 1 bit.

Defining $A$ and $B$ as $(n+1)$-bit integers, where $-Q<A<Q,-Q<B<Q$ and $2^{n-1} \leq Q<2^{n}$, the modular addition (3.1) consists of two steps. the first step is to calculate the sum of the operands (i.e. $A+B$ ). The second step is to perform the modular reduction by comparing the sum $A+B$ with $Q \&-Q$, and adding either $Q$ or $-Q$. Hence, $C$ is defined by:

$$
C \equiv(A+B) \bmod Q
$$

\section{The First Step: SDR Addition Algorithm}

The SDR addition is done in two steps, the first step aims to calculate the partial sum and partial carry as in Table 3.1a. The second step is to perform the carry-free addition as in Table 3.1b. The output of this addition is $(n+2)$-bit long since inputs are $(n+1)$-bit long.

$$
S_{(n+2) \text {-bit }}=A_{(n+1) \text {-bit }}+B_{(n+1) \text {-bit }}
$$

where $S$ is the arithmetic sum of $A$ and $B$.

\section{The Second Step: Comparing And Adding}

The reduction step starts with comparing the most three bits of $S$ with $Q$, if $2^{2} s_{n+2}+$ $2^{1} s_{n+1}+2^{0} s_{n}>0$ then add $-Q$, if $2^{2} s_{n+2}+2^{1} s_{n+1}+2^{0} s_{n}<0$ then add $Q$, else (i.e. $\left.2^{2} s_{n+2}+2^{1} s_{n+1}+2^{0} s_{n}=0\right)$ the output is ready (reduced). 


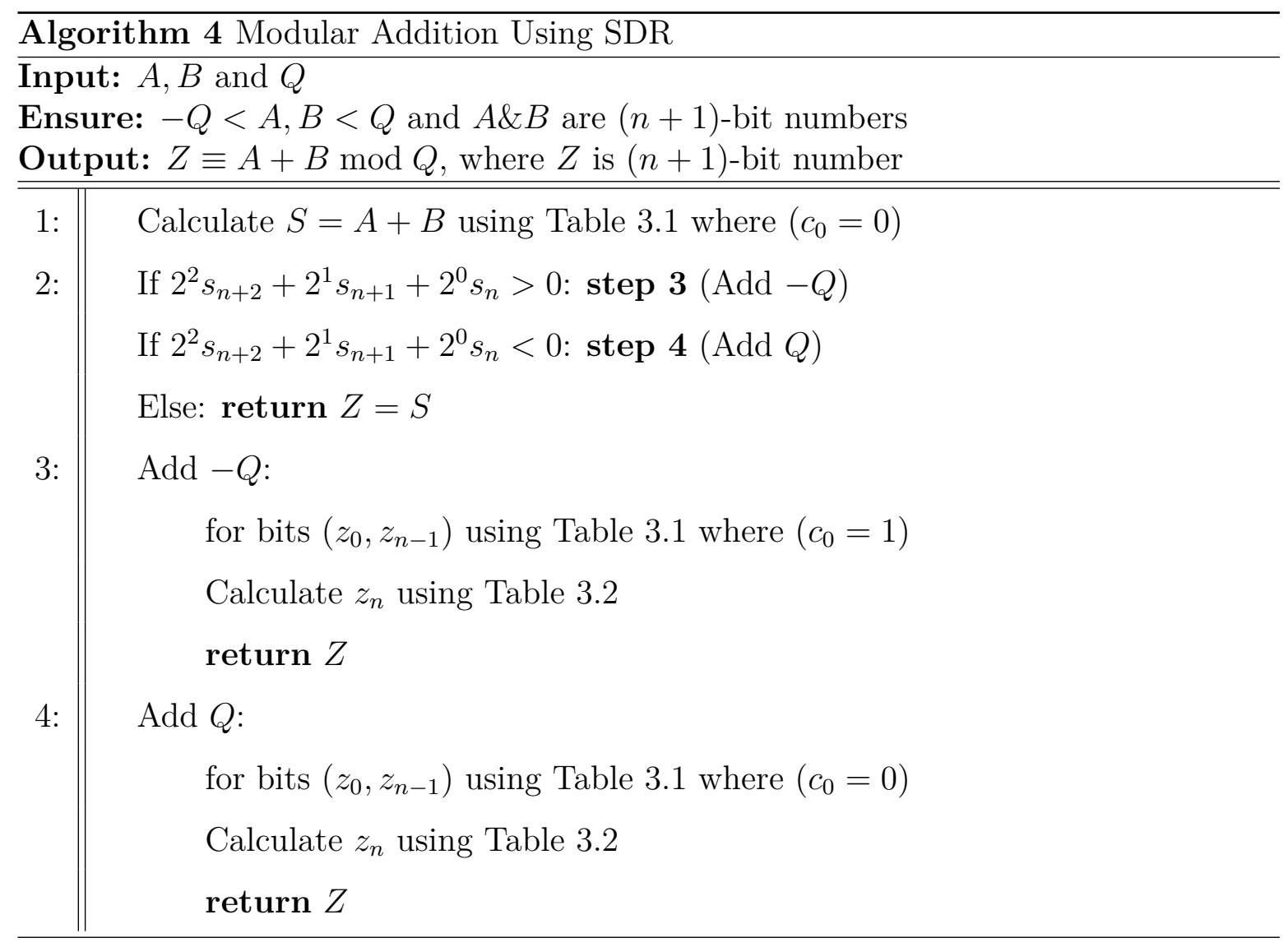

To add $-Q$, since $2^{n-1} \leq Q<2^{n}$ is of the form $\left\{1 q_{n-2} q_{n-3} \ldots q_{0}\right\}$, a simple negative form is targeted to simplify the last addition step, thus,

$$
\begin{aligned}
-Q-1 & =\left\{10 \bar{q}_{n-2} \bar{q}_{n-3} \ldots \bar{q}_{0}\right\} \quad \text { where } \\
\bar{q}_{i} & = \begin{cases}1 & \text { if } q_{i}=0 \\
0 & \text { if } q_{i}=1 .\end{cases}
\end{aligned}
$$

and when adding $-Q-1$, the partial carry first bit should be 1 (i.e. $c_{0}=1$ ).

Hence, the positive $Q$ and the least significant $(n-1)$ bits of the negative version of $Q$ consist only of $(1,0)$. Therefore, the addition rule for this step is simpler than step 1 in Algorithm 4, Table 3.2.

Figure 3.1 shows two examples of the proposed modular addition using SDR. The upper example uses $-Q$ where the carry input is 1 . However, the lower example uses $Q$ where the carry input is 0 . 
Table 3.1: SDR Addition Calculations

\begin{tabular}{|c|c||c|c|}
\hline$a_{k}+b_{k}$ & $a_{k-1}+b_{k-1}$ & $u_{k}$ & $c_{k+1}$ \\
\hline \hline $0+0$ & - & 0 & 0 \\
\hline $1+1$ & - & 0 & 1 \\
\hline$\overline{1}+\overline{1}$ & - & 0 & $\overline{1}$ \\
\hline $1+\overline{1}$ & - & 0 & 0 \\
\hline $0+1$ & $\overline{1}+\overline{1}$ or $0+\overline{1}$ & 1 & 0 \\
\hline $0+1$ & otherwise & $\overline{1}$ & 1 \\
\hline$\overline{1}+0$ & $1+1$ or $1+0$ & $\overline{1}$ & 0 \\
\hline$\overline{1}+0$ & otherwise & 1 & $\overline{1}$ \\
\hline
\end{tabular}

(a) SDR Partial Sum/Carry

\begin{tabular}{|c|c||c|}
\hline$u_{k}$ & $c_{k}$ & $s_{k}$ \\
\hline \hline 0 & 0 & 0 \\
\hline 1 & 0 & 1 \\
\hline 0 & 1 & 1 \\
\hline$\overline{1}$ & 0 & $\overline{1}$ \\
\hline 0 & $\overline{1}$ & $\overline{1}$ \\
\hline$\overline{1}$ & 1 & 0 \\
\hline 1 & $\overline{1}$ & 0 \\
\hline
\end{tabular}

(b) SDR Total Sum

\begin{tabular}{|c|c|c|c|c|c|c|c|c|c|c|c|c|c|c|}
\hline augend $A$ & & & 0 & 0 & 1 & 0 & $\overline{1}$ & 1 & 0 & 1 & 0 & & & \\
\hline addend $B$ & + & & 1 & 0 & $\overline{1}$ & $\overline{1}$ & $\overline{1}$ & 0 & 1 & $\overline{1}$ & 1 & & 0 & \\
\hline$S$ & & 1 & $\overline{1}$ & 0 & $\overline{1}$ & 0 & 1 & $\overline{1}$ & 1 & 1 & $\overline{1}$ & & & \\
\hline$-Q$ & + & & 1 & 0 & 1 & 1 & 0 & 1 & 0 & 0 & 1 & L & 1 & (1) \\
\hline $\bmod \operatorname{sum} C$ & & & 0 & 0 & 1 & 0 & $\overline{1}$ & 1 & 0 & $\overline{1}$ & 1 & {[} & 0 & \\
\hline augend $A$ & & & 0 & 0 & 1 & 0 & $\overline{1}$ & 1 & 0 & 1 & 0 & ) & ( & \\
\hline addend $B$ & + & & $\overline{1}$ & 0 & 1 & 1 & 1 & 0 & $\overline{1}$ & 1 & $\overline{1}$ & ) & ( & \\
\hline$S$ & & 0 & $\overline{1}$ & 1 & 0 & 1 & 0 & 1 & 0 & 0 & $\overline{1}$ & ) & ( & \\
\hline$Q$ & + & & 0 & 1 & 0 & 0 & 1 & 0 & 1 & 1 & 0 & & ( & (0) \\
\hline $\bmod \operatorname{sum} C$ & & & 0 & 0 & 1 & 0 & 0 & 0 & 0 & $\overline{1}$ & $\overline{1}$ & ) & & \\
\hline
\end{tabular}

Figure 3.1: SDR Modular Addition Examples
(1176) step 1 (alg. 4)

step 3 (alg. 4)

(24) step 1 (alg. 4)

step 4 (alg. 4) 
Table 3.2: SDR Modular Addition's Modular Reduction Step

\begin{tabular}{|c||c|c|}
\hline$s_{k}+q_{k}$ & $u_{k}$ & $c_{k+1}$ \\
\hline \hline $0+0$ & 0 & 0 \\
\hline $1+1$ & 0 & 1 \\
\hline $0+1$ & $\overline{1}$ & 1 \\
\hline $1+0$ & $\overline{1}$ & 1 \\
\hline$\overline{1}+0$ & $\overline{1}$ & 0 \\
\hline
\end{tabular}

\begin{tabular}{|c|c||c|}
\hline$u_{k}$ & $c_{k}$ & $s_{k}$ \\
\hline \hline 0 & 0 & 0 \\
\hline 1 & 0 & 1 \\
\hline 0 & 1 & 1 \\
\hline$\overline{1}$ & 0 & $\overline{1}$ \\
\hline$\overline{1}$ & 1 & 0 \\
\hline
\end{tabular}

(a) Reduced Form of SDR Partial Sum/Carry

(b) Reduced Form of SDR Total Sum

\begin{tabular}{|c|c||c|c|c|}
\multicolumn{4}{c|}{$z_{n}$} \\
\hline \multirow{2}{*}{$s_{n+1}$} & \multirow{2}{*}{$s_{n}$} & \multicolumn{3}{|c|}{$s_{n-1}$} \\
\cline { 3 - 5 } & & $\overline{1}$ & 0 & 1 \\
\hline \hline \multirow{2}{*}{$\overline{1}$} & 0 & - & $\overline{1}$ & $\overline{1}$ \\
\cline { 2 - 5 } & 1 & $\overline{1}$ & \multicolumn{2}{|c|}{0} \\
\hline \multirow{3}{*}{0} & $\overline{1}$ & $\overline{1}$ & \multicolumn{2}{|c|}{0} \\
\cline { 2 - 5 } & 0 & \multicolumn{3}{|c|}{0} \\
\cline { 2 - 5 } 1 & 1 & \multicolumn{2}{|c|}{0} & 1 \\
\hline \multirow{2}{*}{1} & $\overline{1}$ & \multicolumn{2}{|c|}{0} & 1 \\
\cline { 2 - 5 } & 0 & \multicolumn{2}{|c|}{1} & - \\
\hline
\end{tabular}

(c) At The Most Two Significant Bits 


\subsection{Modular Multiplier Using SDR}

Modular multiplication is the key component in RNSMM. Algorithm 3 shows multiple instances of using modular multiplier (i.e. steps 1, 2, 4 and 5, within channels of the moduli set). Most of modular multipliers found in literature deal with general modular multipliers (i.e. not specifically for RNS). Some recent designs for RNS are based on general multiplier designs and a modular reduction aftermath. (Asif \& Kong, 2017) who introduced a simple, yet, fast method of modular multiplication that depends on a regular multiplier with radix-4 booth recoding and a Barrett reduction.

The proposed design, intends to do the modular multiplication with a faster or equal speed than the current designs. However, as will be introduced in Section 3.3, base extension of this design outperforms any existing base extension design published so far.

In this research, two designs are developed for this operation, one is based on "division-during-multiplication" technique, and the other is based on "divisionafter-multiplication" (i.e. Barrett reduction). The difference between these two designs is that the division-during-multiplication the output does not need extra calculations. However, division-after-multiplication needs some extra calculations because of the approximation technique used.

\subsubsection{Division-During-Multiplication}

The key component of this method is the SDR Modular Addition that was developed in the previous section (3.1). The modular reduction is applied through distributing the modulo operation over the partial products following the property of the modulo 
operation that:

$$
\begin{aligned}
c & \equiv(a+b) \bmod m \\
c & \equiv(a \bmod m+b \bmod m) \bmod m \\
c & \left.\equiv|| a\right|_{m}+\left.|b|_{m}\right|_{m}
\end{aligned}
$$

where $a, b$ and $c$ are $(\mathrm{n}+1)$ bits long and $m$ is the modulus with (n) bits long.

In this research, a technique called parallel-series algorithm was developed. This algorithm consists of two main operations that run in parallel, and within each operation the process is done serially.

Once the inputs are provided, two operations start. The first operation is the multiplier recoding that is described in Table 3.3, and at the same time the doubling (i.e. modular multiplication by 2) starts as shown in Figure 3.2.

Recoding the multiplier is done in two steps of fixed-complexity. The first is calculating the partial sum and the partial carry of each couples of the multiplier. The second step is to do a carry-free addition. Therefore, the output (i.e. the recoded multiplier) has the length of:

$$
w_{\hat{b}}=\left\lceil\frac{w_{b}}{2}\right\rceil+1
$$

where $w_{\hat{b}}$ is the width of the recoded multiplier, $w_{b}$ is the width of the multiplier. This ensure that the first two inputs of the accumulation step in Figure 3.2 would be ready together. Under those circumstances, the critical path of this approach equals:

$$
\begin{aligned}
\text { Parallel-Series Delay }= & (\text { Delay of Multiplier Recoder }) \\
& +(\text { Delay of ModAdd }) \times\left\lceil\frac{n}{2}\right\rceil
\end{aligned}
$$

\subsubsection{Division-After-Multiplication}

The division-after-multiplication is being achieved using the Improved Barrett Reduction (Improved Barrett Reduction (IBR)) that was developed by Dhem in (Dhem, 1994, 


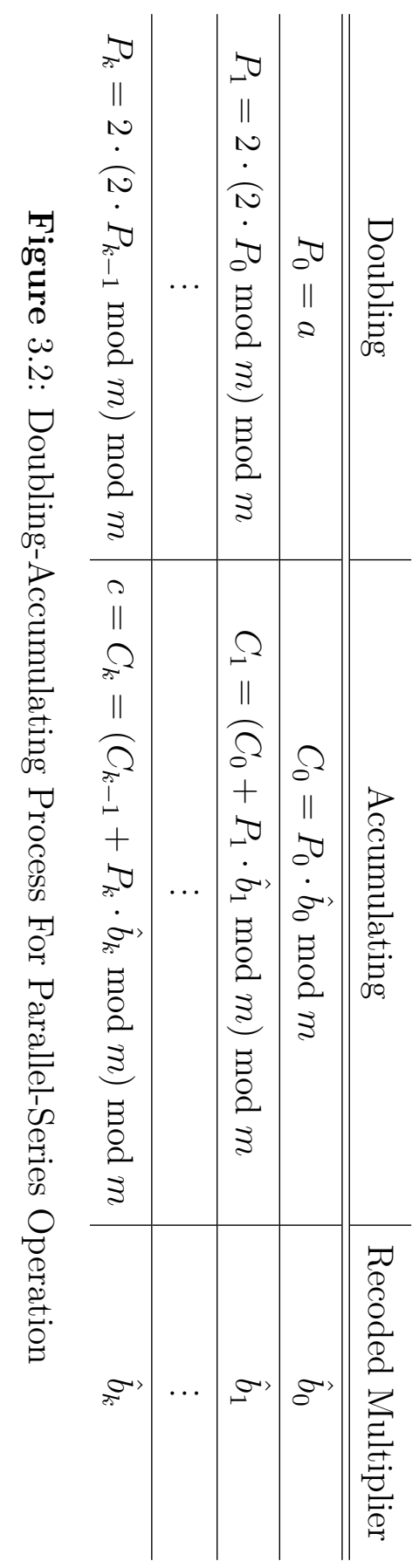


Algorithm 5 Parallel-Series Modular Multiplication Using SDR

Input: $a, b \& m$

Ensure: $a \& b$ are $(n+1)$ bits

Output: $c \equiv a \cdot b \bmod m$

1: $\quad$ Recoding the multiplier $b$ using Table 3.3

2: $\quad$ Perform the doubling-accumulating process depicted in Figure 3.2

Table 3.3: SDR Multiplier Recoding Rules

\begin{tabular}{|c|c|c|c|c|}
\hline \multicolumn{5}{|c|}{$c_{j+1}, u_{j}$} \\
\hline$b_{2 j+1} \backslash b_{2 j}$ & $\overline{1}$ & \multicolumn{2}{|c|}{0} & 1 \\
\hline$\overline{1}$ & $\overline{1}, 1$ & ${ }^{*} 0, \overline{2}$ & $\overline{1}, 2$ & $0, \overline{1}$ \\
\hline 0 & $0, \overline{1}$ & \multicolumn{2}{|c|}{0,0} & 0,1 \\
\hline 1 & 0,1 & ${ }^{*} 1, \overline{2}$ & 0,2 & $1, \overline{1}$ \\
\hline
\end{tabular}

(a) Partial Sum, Carry for Modular Recoding

\begin{tabular}{|c||c|c|c|}
\multicolumn{3}{c}{} & $\hat{b}_{j}$ \\
\hline$u_{j} \backslash c_{j}$ & $\overline{1}$ & 0 & 1 \\
\hline \hline$\overline{2}$ & - & $\overline{2}$ & $\overline{1}$ \\
\hline$\overline{1}$ & $\overline{2}$ & $\overline{1}$ & 0 \\
\hline 0 & $\overline{1}$ & 0 & 1 \\
\hline 1 & 0 & 1 & 2 \\
\hline 2 & $\overline{1}$ & 2 & - \\
\hline
\end{tabular}

(b) Recoded Digit of Multiplier in SDR

1998), which is an improved version of Barrett Reduction (Barrett Reduction (BR)) (Barrett, 1987). First, Barrett suggested the calculation of Modular Reduction to be as follows:

$$
s \bmod m=s-q \cdot m
$$

where,

$$
q=\left\lfloor\frac{\frac{s}{2^{n+v}} \cdot \frac{2^{n+u}}{m}}{2^{u-v}}\right\rfloor
$$

where $s$ is the input, $m$ is the modulus, $q$ is the quotient, $n$ is the width of both $s$ and $m$ and $u$ and $v$ are some constants.

Hence, the calculation of the quotient $q$ transformed into a multiplication with precalculated one-time value $\left(\frac{2^{n+u}}{m}\right)$ and a shift operation. Thus, (Dhem, 1994) suggested to modify the calculation of the quotient $q$ to be as Equation 3.9 and in order to eliminate 
the error. The values of $u$ and $v$ are $n+3$ and -2 respectively.

$$
\hat{q}=\left\lfloor\frac{\left\lfloor\frac{s}{2^{n-2}}\right\rfloor \cdot\left\lfloor\frac{2^{2 n+3}}{m}\right\rfloor}{2^{n+1}}\right\rfloor
$$

The sequence in this approach is as the follows:

$$
\begin{aligned}
s & =a \times b \\
z & =s \bmod m \\
z & =s-q \cdot m \\
q=\hat{q} & =\left\lfloor\frac{\left\lfloor\frac{s}{2^{n-2}}\right\rfloor \cdot\left\lfloor\frac{2^{2 n+3}}{m}\right\rfloor}{2^{n+1}}\right\rfloor
\end{aligned}
$$

Thus, this approach has three SDR multiplication as in equations 3.10, 3.12 and 3.13, and a single SDR addition (subtraction) as in equation 3.12.

As shown in Algorithm 6, the multiplication in this approach starts with multiplier recoding following Table 3.3. This is followed by generating partial products array, according to the rule described in Table 3.4. This partial products array is fed into an SDR addition tree to generate their summation (see Figure 3.1). The key element of

this tree is the SDR addition element described in Table 3.1 and shown in Figure 3.4. Hence, the depth of this tree is:

$$
\mathrm{SDR} \text { reduction tree depth }=\left\lceil\log _{2}\left(\left\lceil\frac{n}{2}\right\rceil+1\right)\right\rceil
$$

\subsection{Base Extension Using SDR}

Montgomery Multiplication of Algorithm 3, needs two base extension operations. The idea behind base extension is to re-construct the number from its RNS into conventional binary and convert it back into an auxiliary coprime moduli set. In this research, a new base extension design using SDR is introduced. This design is based on Chinese 

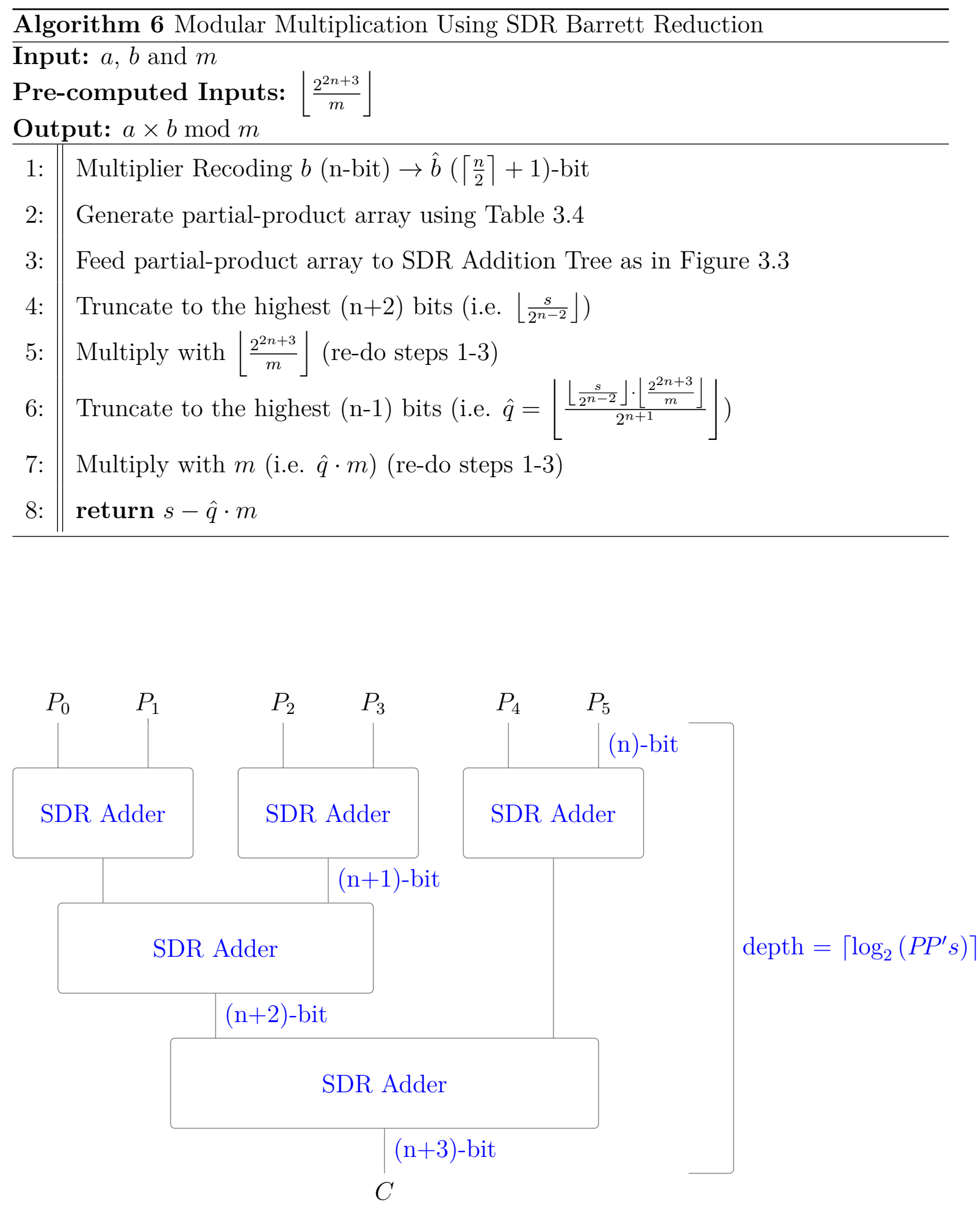

Figure 3.3: SDR Addition Tree Example. 
Table 3.4: SDR Recoded Multiplier Multiplication Rule

\begin{tabular}{|c|c|}
\hline$\hat{b}$ & Partial Product if Multiplicand $=a$ \\
\hline \hline 2 & $a<<1$ \\
1 & $a$ \\
0 & 0 \\
$\overline{1}$ & $\bar{a}($ i.e. $\overline{(\overline{1})}=1, \overline{(1)}=\overline{1})$ \\
$\overline{2}$ & $\bar{a}<<1$ \\
\hline
\end{tabular}

$a$ (n bits) $b$ (n bits)

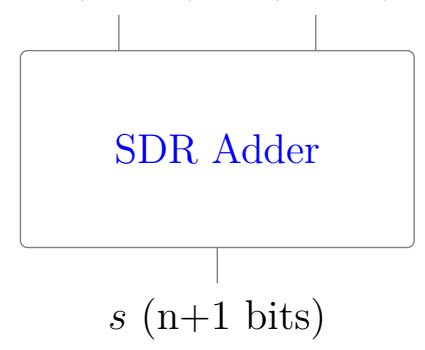

Figure 3.4: SDR Addition Element

Remainder Theorem. In the following subsection, the mathematical definition of the Chinese Remainder Theorem (CRT) and the Mixed-Radix Conversion are introduced and differentiated.

\subsubsection{CRT vs MRC}

As introduced in section 1.4.1, any integer $X \in[0, M-1]$ represented in a moduli set has the following RNS representation $X=\left\{x_{1}, x_{2}, \cdots, x_{N}\right\}$, can be converted into binary using the CRT as follows:

$$
\begin{aligned}
X & =\left.\left.\left|\sum_{1}^{N}\right| x_{i} M_{i}^{-1}\right|_{m_{i}} M_{i}\right|_{M} \\
\text { or, } X & =\sum_{1}^{N}\left|x_{i} M_{i}^{-1}\right|_{m_{i}} M_{i}-\alpha \cdot M
\end{aligned}
$$

for simplicity let's define

$$
\xi_{i}=\left|x_{i} M_{i}^{-1}\right|_{m_{i}}
$$


where $\alpha$ is the correction factor that can be computed using a redundant modulo (Shenoy \& Kumaresan, 1989) (let's name it $m_{x}$ ). To simplify the calculation of this correction factor, this redundant modulo can have the form of $2^{k}$. Thus, modular computation of this redundant channel is simple and straight forward (i.e. $|A|_{2^{k}}=$ the least significant $k$ bits) as shown in equation 3.20. It is notable that $\alpha$ is bounded by the number of moduli in the moduli set " $N$ " (i.e. $0 \leq \alpha<N$ ). Therefore, the width of this channel can be reduced to include only the number of moduli " $N$ " (i.e. $\alpha=2^{k}$, $\left.k=\left\lceil\log _{2}(N)\right\rceil\right)$. Since,

$$
\begin{aligned}
X & =\sum_{1}^{N} \xi_{i} M_{i}-\alpha \cdot M \\
|X|_{m_{x}} & =\left|\sum_{1}^{N} \xi_{i} M_{i}-\alpha \cdot M\right|_{m_{x}}
\end{aligned}
$$

re-arranging 3.18;

$$
\begin{aligned}
|\alpha \cdot M|_{m_{x}} & =\left.\left.\left|\sum_{1}^{N} \xi_{i} M_{i}-\right| X\right|_{m_{x}}\right|_{m_{x}} \\
\alpha & =\left.|| M\right|_{m_{x}} ^{-1} \cdot \sum_{1}^{N} \xi_{i} M_{i}-\left.|X|_{m_{x}}\right|_{m_{x}}
\end{aligned}
$$

According to (Huang, 1983), to convert a number $X$ represented in RNS, to its binary equivalent using $\mathrm{MRC}$, then:

$$
X=x_{1}^{\prime}+u_{2} x_{2}^{\prime}+\cdots+u_{N} x_{N}^{\prime}
$$

where $u_{2}=m_{1}, u_{3}=m_{1} m_{2}, u_{4}=m_{1} m_{2} m_{3}$, and so forth. $x_{i}^{\prime}$ s are computed as follows:

$$
\begin{aligned}
x_{1}^{\prime} & =x_{1} \\
x_{2}^{\prime} & =\left.\left.\left|\left(x_{2}-x_{1}^{\prime}\right)\right| m_{1}^{-1}\right|_{m_{2}}\right|_{m_{2}} \\
x_{3}^{\prime} & =\left.\left.\left|\left(\left(x_{3}-x_{1}^{\prime}\right)\left|m_{1}^{-1}\right|_{m_{3}}-x_{2}^{\prime}\right)\right| m_{2}^{-1}\right|_{m_{3}}\right|_{m_{3}} \\
& \vdots \\
x_{N}^{\prime} & =\left.\left.\left|\left(\cdots\left(x_{N}-x_{1}^{\prime}\right)\left|m_{1}^{-1}\right|_{m_{n}}-\cdots-x_{N-1}^{\prime}\right)\right| m_{N-1}^{-1}\right|_{m_{N}}\right|_{m_{N}}
\end{aligned}
$$


where $\left|m_{i}^{-1}\right|_{m_{j}}$ is the multiplicative inverse of $m_{i}$ modulo $m_{j}$ (i.e. $m_{i} \cdot\left|m_{i}^{-1}\right|_{m_{j}} \equiv$ $1 \bmod m_{j}$ ). Therefore, the MRC gets rid of calculating the correction factor in CRT that is computed in equation 3.20 (Schinianakis \& Stouraitis, 2013).

When using the CRT all the terms $\xi_{i} M_{i}$ need be ready at the same time, and can be fed directly into the addition tree. The calculation of the correction factor is easily done since it depends on the redundant modulo $m_{x}$ which will be of the form of $2^{k}$. Hence, the calculation of the correction factor is just another addition term that is fed to the addition tree. Note that the term $-\alpha \cdot M$ is being pre-computed and stored in a Read Only Memory (ROM). Hence, the $\alpha$ value is used as an address to the value $-\alpha \cdot M$.

On the other hand, in MRC the computation of $x_{i}^{\prime \prime}$ s is performed serially, where $x_{i}^{\prime}$ depends on the values of $x_{i-1}^{\prime}, x_{i-2}^{\prime}$ and so forth. This has a linear complexity (its complexity is $\mathcal{O}(N)$, where $N$ is the number of moduli in the RNS moduli set).

The proposed design, for base extension operation using SDR, utilizes the CRT with a redundant modulo $\left(m_{x}\right)$ of the form of $2^{k}$ where $k=\left\lceil\log _{2}(N)\right\rceil$ where $N$ is the number of moduli in the RNS moduli set. This redundant modulus is exploited to calculate the correction factor (see equation 3.16) in the CRT (see equation 3.20).

The number will be converted from base moduli set $M_{\text {set }}$ into an auxiliary moduli set $\widetilde{M}_{\text {set }}$. As in Figure 3.5, the first operation is the calculation of the terms $\xi_{i}$ 's. These terms are calculated through a modular multiplication that was developed in the previous section, and the moduli of this operation are the elements of the base moduli set (i.e. $M_{\text {set }}:=\left\{m_{1}, m_{2}, \cdots, m_{N}\right\}$ ). The second operation is the modular multiplication $\left(\left|\xi_{i} M_{i}\right|_{\widetilde{m}_{j}}\right)$ where the moduli of this step are the moduli of the auxiliary moduli set (i.e. $\widetilde{M}_{\text {set }}:=\left\{\widetilde{m}_{1}, \widetilde{m}_{2}, \cdots, \widetilde{m}_{N}\right\}$ ).

The main operation after the second modular multiplication is Modular Addition tree as Figure 3.3. However, the element of this tree is the modular addition that is 
described in section 3.1. The depth of this tree is:

$$
\operatorname{depth}=\left\lceil\log _{2}(N+1)\right\rceil
$$

where $N$ is the number of moduli in the base moduli set (i.e. $M_{\text {set }}$ ). Thus, the complexity of this operation is two modular multiplication operations and an SDR Modular Addition Tree:

$$
\begin{aligned}
\text { SDR Base Extension Complexity }= & 2 \times(\text { SDR ModMult }) \\
& +(\text { SDR ModAdd }) \times\left(\left\lceil\log _{2}(N+1)\right\rceil\right)
\end{aligned}
$$

\begin{tabular}{c|cccc}
$M_{s e t} \backslash \widetilde{M}_{s e t}$ & $\widetilde{m}_{1}$ & $\widetilde{m}_{2}$ & $\cdots$ & $\widetilde{m}_{N}$ \\
\hline$m_{1}$ & $\left|\xi_{1} M_{1}\right|_{\widetilde{m}_{1}}$ & $\left|\xi_{1} M_{1}\right|_{\widetilde{m}_{2}}$ & $\cdots$ & $\left|\xi_{1} M_{1}\right|_{\widetilde{m}_{N}}$ \\
$m_{2}$ & $\left|\xi_{2} M_{2}\right|_{\widetilde{m}_{1}}$ & $\left|\xi_{2} M_{2}\right|_{\widetilde{m}_{2}}$ & $\cdots$ & $\left|\xi_{2} M_{2}\right|_{\widetilde{m}_{N}}$ \\
$\vdots$ & $\vdots$ & $\vdots$ & $\ddots$ & $\vdots$ \\
$m_{N}$ & $\left|\xi_{N} M_{N}\right|_{\widetilde{m}_{1}}$ & $\left|\xi_{N} M_{N}\right|_{\widetilde{m}_{2}}$ & $\cdots$ & $\left|\xi_{N} M_{N}\right|_{\widetilde{m}_{N}}$ \\
$|-\alpha M|_{\widetilde{m}_{j}}$ & $|-\alpha M|_{\widetilde{m}_{1}}$ & $|-\alpha M|_{\widetilde{m}_{2}}$ & $\cdots$ & $|-\alpha M|_{\widetilde{m}_{N}}$ \\
\hline$\left|\sum_{1}^{N}(\cdots)\right|_{\widetilde{m}_{j}}$ & $\left|\sum_{1}^{N}(\cdots)\right|_{\widetilde{m}_{1}}$ & $\left|\sum_{1}^{N}(\cdots)\right|_{\widetilde{m}_{2}}$ & $\cdots$ & $\left|\sum_{1}^{N}(\cdots)\right|_{\widetilde{m}_{N}}$
\end{tabular}

Figure 3.5: SDR Base Extension 


\section{Chapter 4 \\ Complexity Analysis}

The analysis of these designs will be held on theoretical side. The comparison will be held on delay using Unit Gate Delay (Unit Gate Delay (UGD)), which equivalent to any primitive gate ( AND, OR). NOT and BUFFER gates are ignored. Hence, the $\mathrm{XOR}$ and the XNOR gates' delay is (2 UGD).To estimate the area Unit Gate Area (Unit Gate Area (UGA)) is used, and as the UGD, NOT and BUFFER are ignored (Zimmermann, 1999).

Moreover, this research is dedicated to have a scalable design, which means that it will be easily implemented to operands of 2048 bits long and more. For the sake of comparison with other designs, the operands in this research is considered to be 2048 bits long. Hence, if the moduli in the RNS set are of the length of 20 bits the number needed is 103 moduli.

\subsection{Complexity Analysis}

Since the deign was divided into three main parts; the analysis will be held on each one of the three elements. From Algorithm 3, the delay of the design is:

$$
\begin{aligned}
\text { delay }= & 4 \times(\text { delay of modular multiplication }) \\
+ & 2 \times(\text { delay of base exntension }) \\
+ & 1 \times(\text { delay of modular addition })
\end{aligned}
$$

The following subsections will represent the design of each part, the base elements of them and their internal designs, and the theoretical analysis of each one. Since the SDR bit has three options $\{\overline{1}, 0,1\}$, two binary bits is used to code a single SDR bit. 
Table 4.1: SDR Encoding

\begin{tabular}{|c|c|}
\hline SDR bit & Binary encoding \\
\hline \hline$\overline{1}$ & 10 \\
\hline 0 & 00 \\
\hline 1 & 01 \\
\hline
\end{tabular}

Table 4.1 shows the encoding used in this research. This encoding has been chosen so that the Most Significant Bit(s) (MSB) in each SDR bit has the negative value, while the Least Significant Bit(s) (LSB) of the SDR bit has the positive value.

Hence, the SDR-to-binary conversion is achieved by separating the positive bits and the negative bits, calculating the one's complement of the negative number and performing a binary addition with carry-in equals to 1. For example, to convert $1 \overline{1} 0101 \overline{1} \overline{1}$ (81) into binary, the the positive number of it is 01010100 and the negative number is 001000011 and the one's complement of the negative number is 110111100 (note the sign bit is added). Performing a binary addition of these two numbers with carry-in of 1 results of $001010001(81)$.

\subsubsection{Complexity of SDR Modular Adder}

The top-down technique is uses in this research to describe the SDR Modular Adder as shown in Figure 4.1. As Section 3.1, the modular adder consists of two main steps. The first step is the SDR addition, and the second step is the modular reduction.

The SDR addition starts with calculating the partial sum and the partial carry. Figure 4.2 represents the logic design of the SDR One-Bit Partial-Sum/Carry (Partial SumCarry (PSC)). Figure 4.3 shows the logical design of the carry-free summation of partial sum and partial carry that were generated by circuit of Figure 4.2. Thus, the 


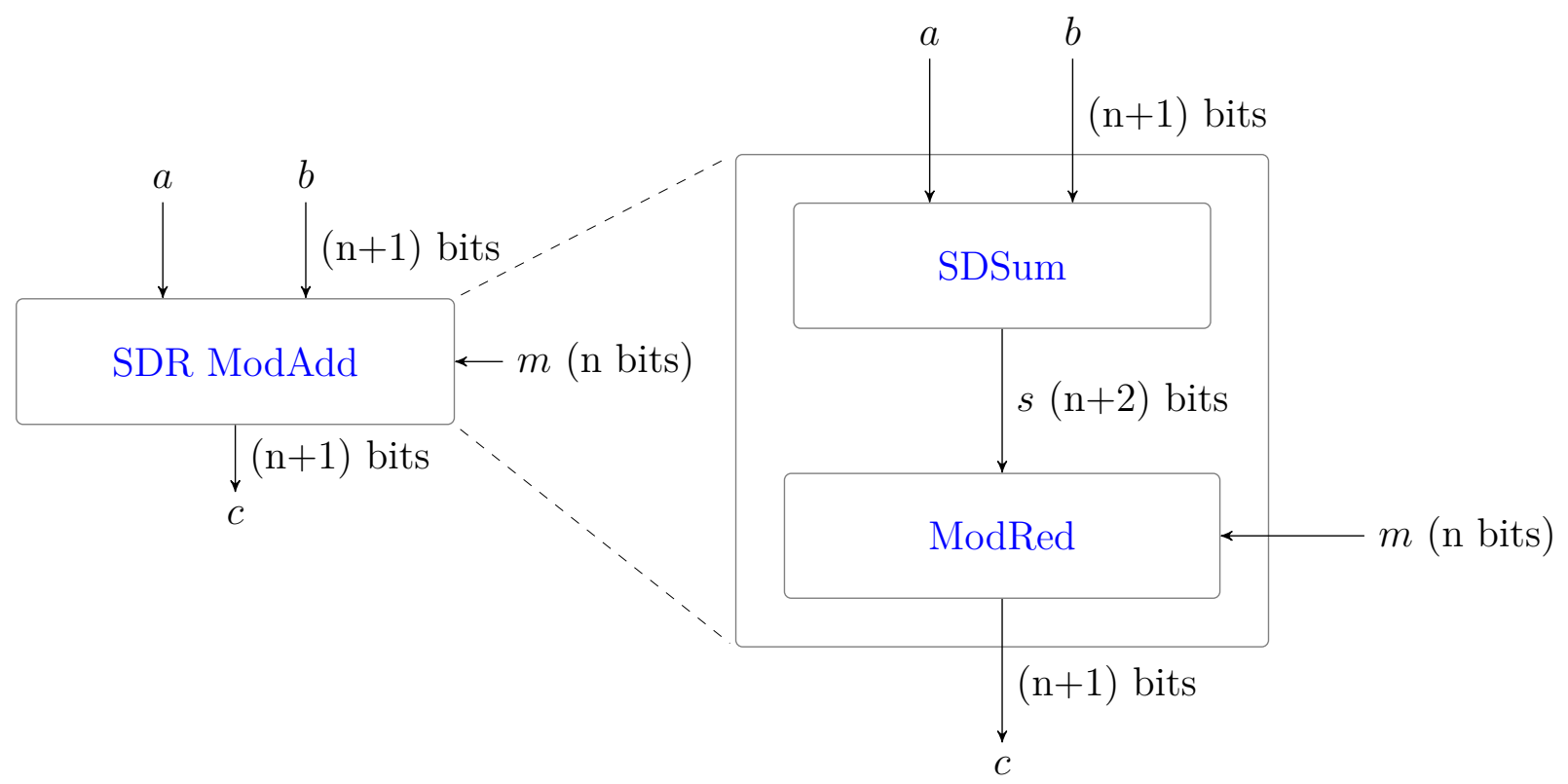

Figure 4.1: SDR Modular Adder

SDSum circuit has the delay of:

$$
\begin{aligned}
\text { Delay }_{\text {SDSum }} & =\text { Delay }_{P S C}+\text { Delay }_{\text {TwoBitSum }} \\
& =5 \mathrm{UGD}+3 \mathrm{UGD} \\
\text { Delay }_{\text {SDSum }} & =8 \mathrm{UGD}
\end{aligned}
$$

As mentioned in Section 3.1, the modular reduction in the SDR Modular adder has a simpler rule for adding the modulus (or the negative of the modulus). The ModRed performs both adding the modulus and the negative of the modulus while the output is passed through the MUX. The select line of the MUX is the result of the comparison of the 3-MSB of the output of SDSum. Figure 4.4 shows the simpler circuit design of a partial sum and partial carry (PSCless) for the ModRed circuit. On the other hand, Figure 4.5 shows the carry-free addition circuit (TwoBitSumless) that adds the output of the previous circuit ( PSCless). The delay of ModRed is as follows:

$$
\begin{aligned}
\text { Delay }_{\text {ModRed }} & =\text { Delay }_{\text {PSCless }}+\text { Delay }_{\text {TwoBitSumless }}+\text { Delay }_{\text {MUX2x1 }} \\
& =4+3+2 \\
\text { Delay }_{\text {ModRed }} & =9 \mathrm{UGD}
\end{aligned}
$$




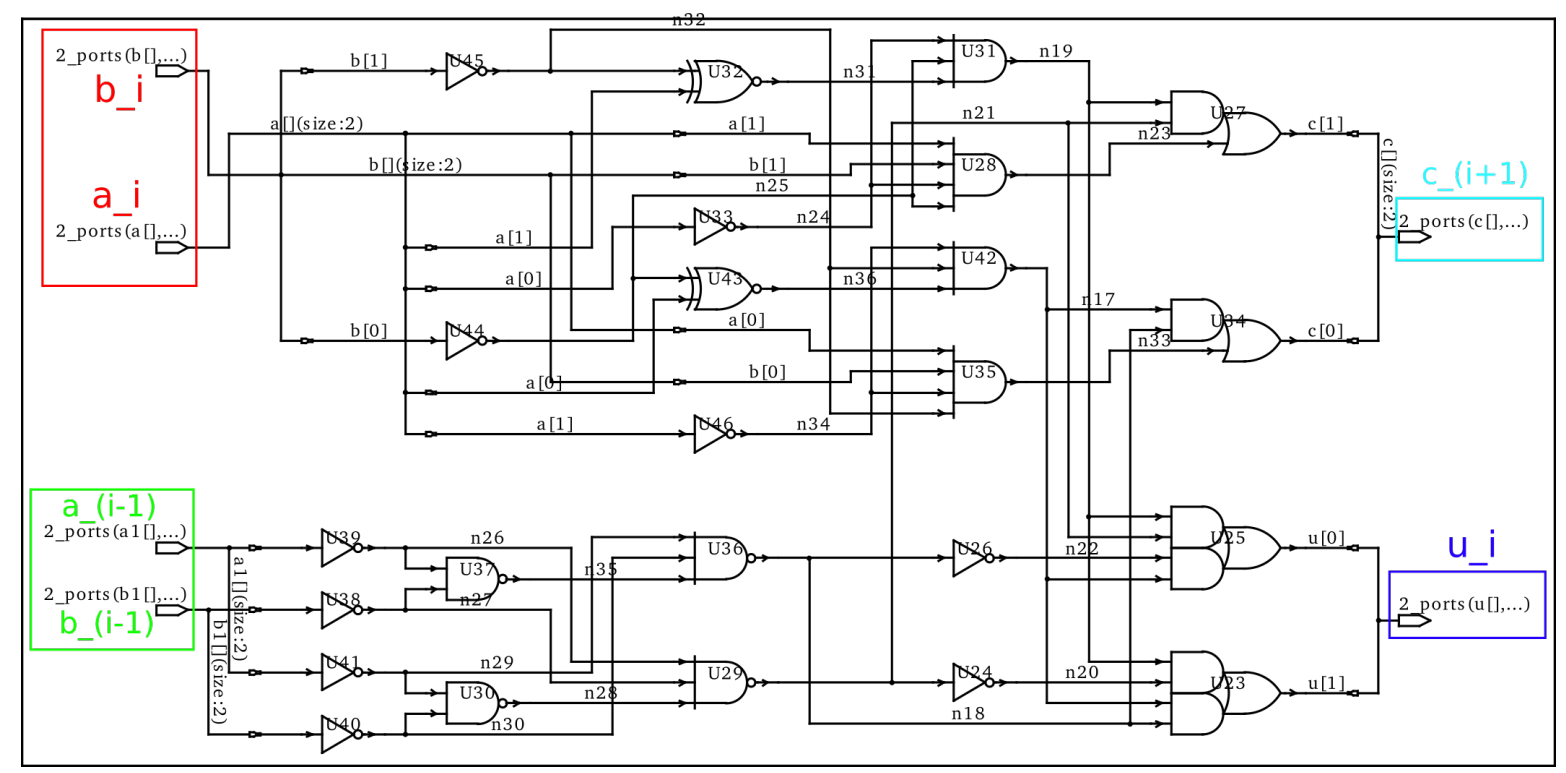

Figure 4.2: SDR One-Bit PSC

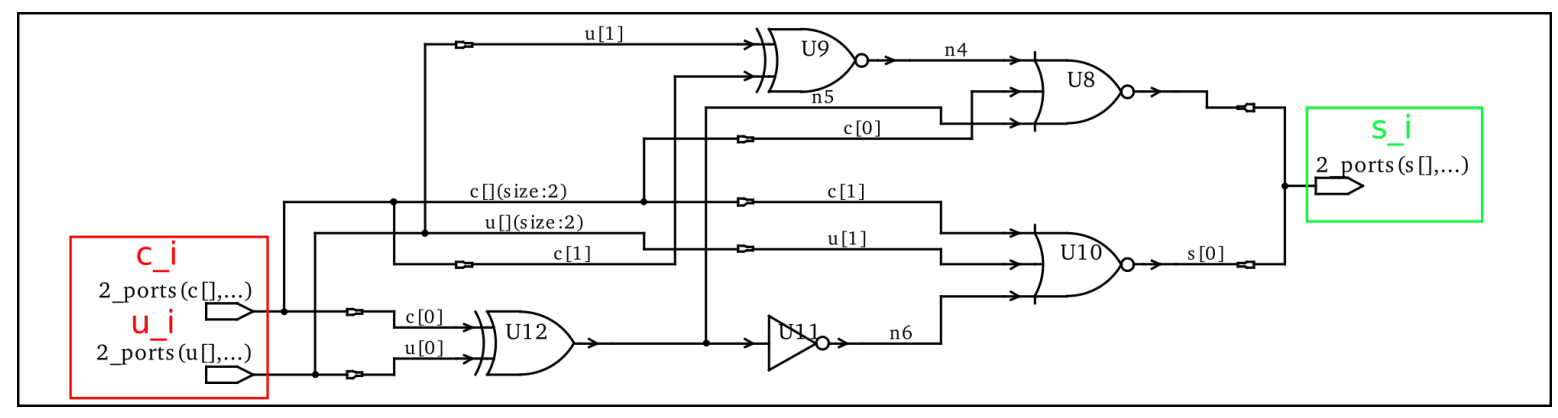

Figure 4.3: SDR PSC TwoBitSum 


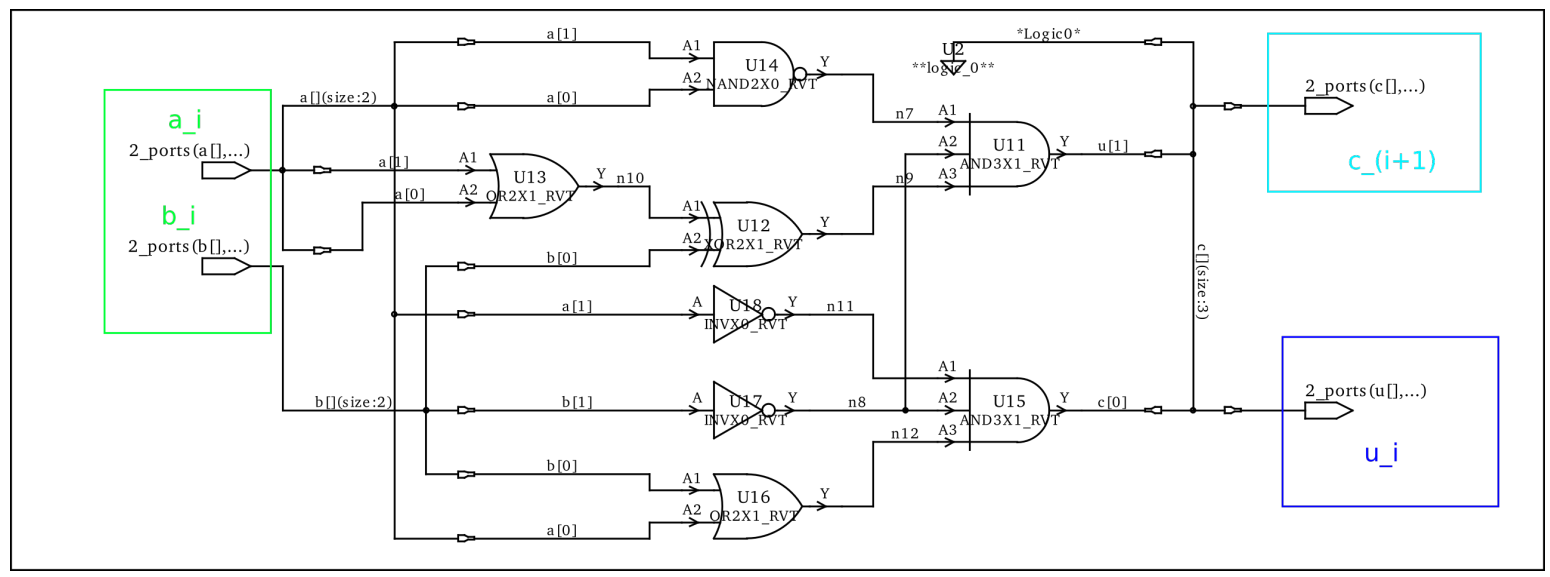

Figure 4.4: SDR PSCless

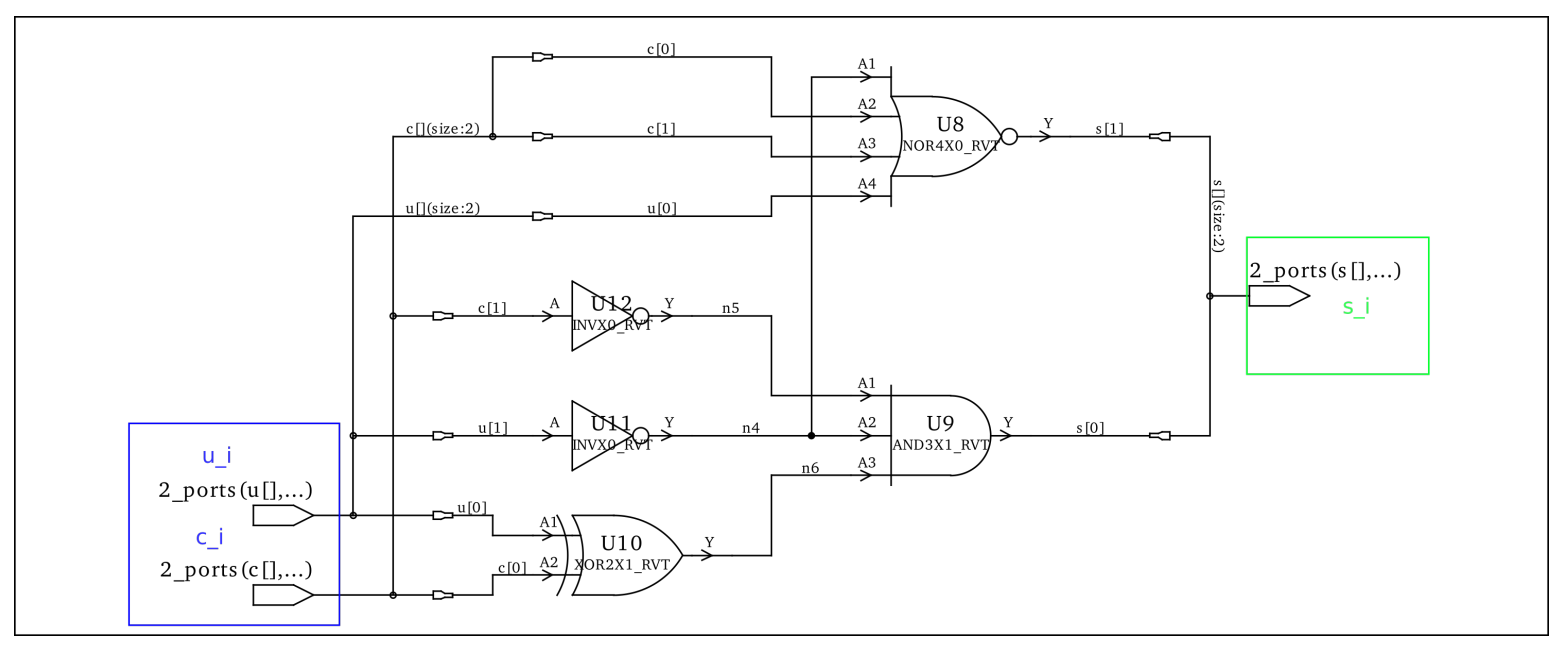

Figure 4.5: SDR TwoBitSumless

The delay of the SDR Addition and Modular addition have a fixed complexity, irrespective of the word length the inputs. This is always the case (the delay cannot be greater than the critical path or the worst case). Hence, the delay for the SDR Modular Adder is 17 UGD.

Recall the schematic of the SDR Modular Adder, the area of different elements area as follows: the area of PSC is 30 UGA, TwoBitSum is 8 UGA, PSCless is 9 UGA, TwoBitSumless is 6 UGA and the comparison is 11 plus $(n+1) \times 4$. Hence, the total area of the SDR Modular Adder where $a$ and $b$ are inputs of the length of $(\mathrm{n}+1)$ and $m$ is the modulus of the length of $n$ and $c$ is the output of the length of $(\mathrm{n}+1)$, the area 
is given by:

$$
\begin{aligned}
\text { Area }_{\text {SDRModAdd }}= & n \times \text { Area }_{P S C} \\
+ & (n+1) \times \text { Area }_{\text {TwoBitSum }} \\
+ & (n-1) \times \text { Area }_{\text {PSCless }} \\
+ & n \times \text { Area }_{\text {TwoBitSumless }} \\
& +11+(n+1) \times 4 \\
= & 30 \cdot n+8 \cdot(n+1)+9 \cdot(n-1)+6 \cdot n+4 \cdot(n+1)+11 \\
\text { Area }_{\text {SDRModAdd }}= & 51 n+14 \mathrm{UGA}
\end{aligned}
$$

\subsubsection{Complexity of SDR Modular Multiplier}

As mentioned earlier, in this research two designs for the modular multiplication process were developed. The first is the "division-during-multiplication", which has the "serialparallel" architecture. The second is the "division-after-multiplication", which is based on the improved Barrett reduction (Dhem, 1994, 1998).

In this subsection, both designs will be implemented and analyzed. It will be shown next that the "division-after-multiplication" is faster than the "division-duringmultiplication". Nevertheless, the "division-during-multiplication" is being studied for its potential in a future improvement.

\section{Division-During-Multiplication}

In this research, this design was divided into three main elements. The first element is the MultRec (i.e. the multiplier recoder) to recode the multiplier into radix-4 coding $(b \rightarrow \hat{b})$. The second element is the Doubler, which performs $\left(P_{i+1}=4 \cdot P_{i} \bmod m\right)$. The third element is the YA that multiply the recoded multiplier digits $\hat{b}_{i}$ with the $P_{i}$ and then performs a SDR Modular Addition using SDR ModAdd (which was developed in the previous subsection). 
Table 4.2: SDR Radix-4 Encoding

\begin{tabular}{|c||c|}
\hline$\hat{b}_{i}$ & Binary Encoding \\
\hline \hline$\overline{2}$ & 100 \\
\hline$\overline{1}$ & 110 \\
\hline 0 & 000 \\
\hline 1 & 010 \\
\hline 2 & 001 \\
\hline
\end{tabular}

MultRec consists of two steps. The first step is recPSC which computes the partial sum and the partial carry of SDR bits of the multiplier (i.e. $b_{2 i}, b_{2 i+1}$ ) following the rule in Table 3.3a. The second step is recTwoDigSum which performs a carry free SDR radix- 4 addition following the rules in Table $3.3 \mathrm{~b}$. Since, the recoded multiplier $(\hat{b})$ has a radix 4, it needs a new encoding. This encoding represents each digit using three binary bits as shown in Table 4.2 .

If the operands of the multiplication have the width of $(n+1)$, the width of the recoded multiplier is:

$$
w_{\hat{b}}=\left\lceil\frac{n+1}{2}\right\rceil+1
$$

Hence, for the a 20-bit channel width, the multiplier and the multiplicand are $(n+1)$ SDR bits, and the width of the recoded multiplier is 12 SDR bits.

Figure 4.6 shows the design of the recPSC which has the delay of 4 UGD and area of 21 UGA. Figure 4.7 shows the design of recTwoDigSum, where the delay is 6 UGD and area is 23 UGA. Hence, the total delay of the MultRec is 10 UGD.

Doubler is just two consecutive ModRed with two left shift (multiplication by 2). The left shift has no cost because it is just a wire connection. Hence, the Doubler has a delay of two ModRed which equals 14 UGD. The depth of the Doubler chain is the width of the recoded multiplier minus one $\left(w_{\hat{b}}-1\right)$. 


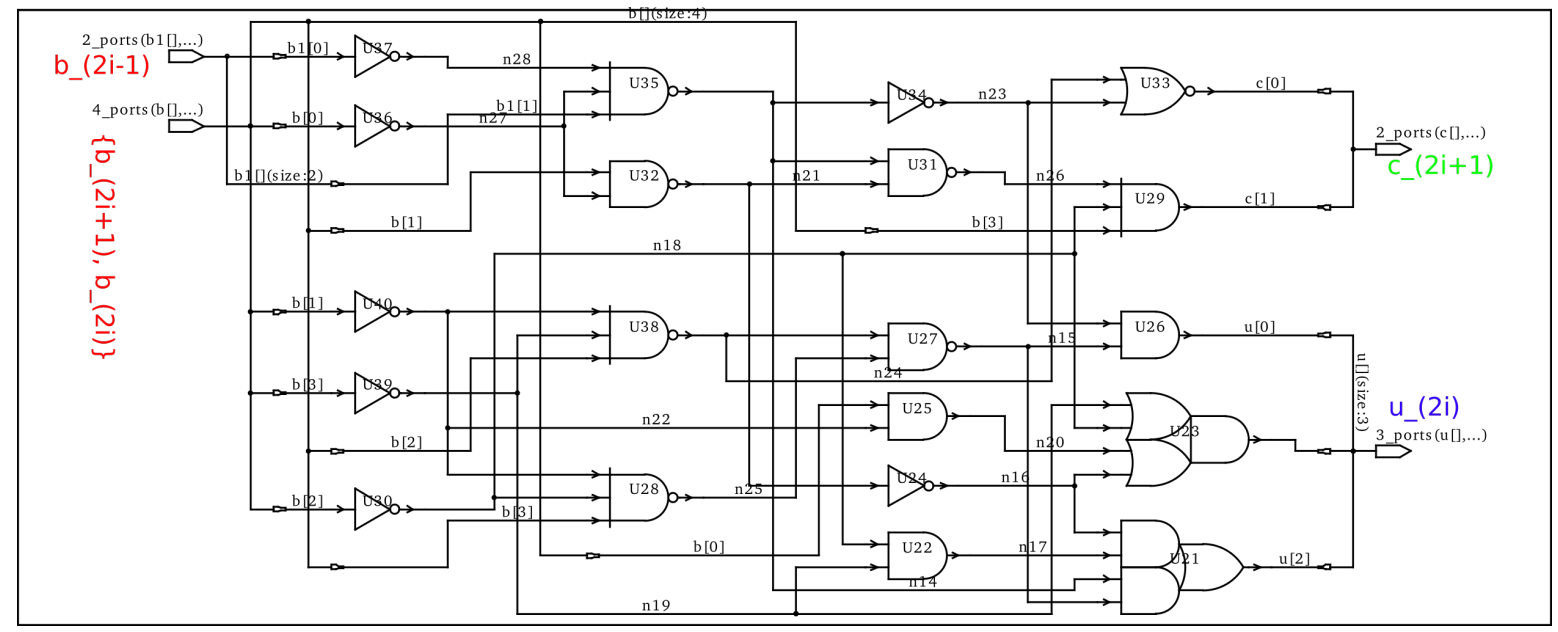

Figure 4.6: SDR recPSC

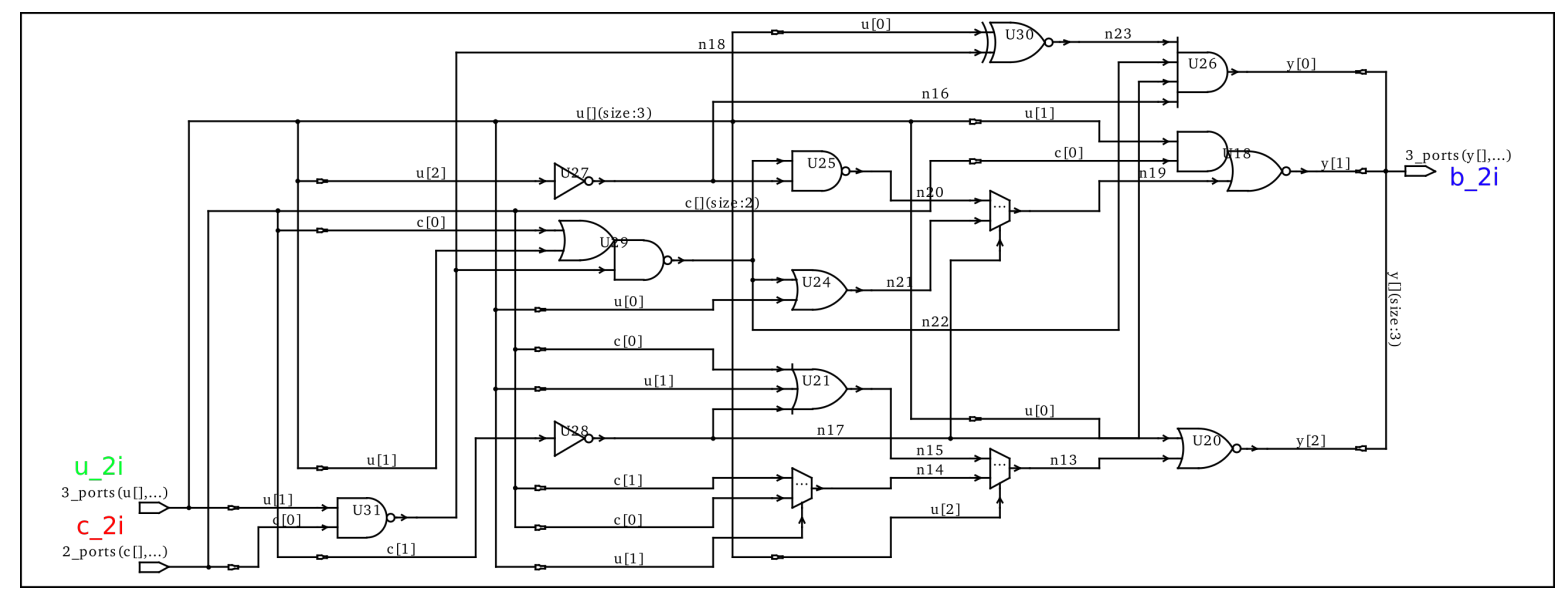

Figure 4.7: SDR recTwoDigSum 
Since, YA is $P_{k} \cdot \hat{b}_{k} \bmod m$ as shown in Figure 3.2. The delay of computing YA is:

$$
\begin{aligned}
& \text { Delay }_{Y A}=\text { Delay }_{\text {SDInv }}+\text { Delay }_{\text {ModRed }} \\
& \text { Delay }_{Y A}=9 \text { UGD }
\end{aligned}
$$

where SDInv is the circuit to find the negative of any SDR number by replace each 1 by $\overline{1}$ and each $\overline{1}$ by 1 and its delay is 2 UGD.

Hence, the accumulator part Figure 3.2 is the critical path for this design. Thus, the delay of this design is:

$$
\text { Delay }_{\text {Division-during-multiplicatoin }}=23+15 \times\left\lceil\frac{n}{2}\right\rceil
$$

\section{Division-After-Multiplication}

As per section 3.2, the "division-after-multiplication" is based on Improved Barrett Reduction that was introduced by (Dhem, 1994, 1998). As in Equation 4.9 and 4.10.

$$
\begin{aligned}
& s \bmod m=s-\hat{q} \cdot m \\
& \hat{q}=\left\lfloor\frac{\left\lfloor\frac{s}{2^{n-2}}\right\rfloor \cdot\left\lfloor\frac{2^{2 n+3}}{m}\right\rfloor}{2^{n+1}}\right\rfloor
\end{aligned}
$$

There are three SDR multiplications, the first is $(s=a \times b)$, the second is $\left(\left\lfloor\frac{s}{2^{n-2}}\right\rfloor \cdot\left\lfloor\frac{2^{2 n+3}}{m}\right\rfloor\right)$, and the third is $\hat{q} \cdot m$. In addition to, an $\operatorname{SDR}$ addition $(s-\hat{q} \cdot m)$ is also needed.

The delay complexity of this design is:

$$
\begin{aligned}
\text { Delay }_{\text {Division-After-Multiplication }=} & 1 \times \operatorname{Delay}_{\text {SDMult }}(n) \\
& +1 \times \operatorname{Delay}_{\text {SDMult }}(n+4) \\
& +1 \times \operatorname{Delay}_{\text {SDMult }}(n+3) \\
& +1 \times \operatorname{Delay}_{\text {SDSum }}(2 n)
\end{aligned}
$$

where SDMult consists of a multiplier recoder (MultRec), an SDR Digit multiplier (XYA) that follows the rules of Table 3.4, and an SDR addition tree as shown in 
Figure 3.3. Hence, the delay of SDMult is:

$$
\begin{aligned}
\text { Delay }_{S D M u l t}= & \text { Delay }_{\text {MultRec }} \\
& + \text { Delay }_{X Y A} \\
& + \text { Delay }_{\text {SDRAdditionTree }}
\end{aligned}
$$

where SDR Addition Tree delay is:

$$
\begin{aligned}
\text { Delay }_{\text {SDRAdditionTree }} & =\text { Delay }_{\text {SDSum }} \times \text { Depth of the Additon tree } \\
& =8 \times\left\lceil\log _{2}\left(w_{\hat{b}}\right)\right\rceil
\end{aligned}
$$

where $w_{\hat{b}}$ is the width of the recoded multiplier (i.e. $\left\lceil\frac{n}{2}\right\rceil+1$ ). Whereas, the delay of XYA is 5 UGD. Note that, the second and the third SDMult do not need recoding to be done at the time of multiplication since the multipliers in both cases (i.e. $\left\lfloor\frac{2^{2 n+3}}{m}\right\rfloor$ and $m$ ) are pre-computed values. Hence:

$$
\begin{aligned}
\text { Delay }_{\text {SDMult }} & =10+5+8 \cdot\left\lceil\log _{2}\left(w_{\hat{b}}\right)\right\rceil \\
\text { Delay }_{\text {SDMult }} & =15+8 \cdot\left\lceil\log _{2}\left(w_{\hat{b}}\right)\right\rceil
\end{aligned}
$$

and

$$
\text { Delay }_{\text {SDMult without recoding }}=5+8 \cdot\left\lceil\log _{2}\left(w_{\hat{b}}\right)\right\rceil
$$

Eventually, the total delay of "division-after-multiplication" approach is:

$$
\begin{aligned}
\text { Delay }_{\text {Division-After-Multiplication }}= & 15+8 \cdot\left\lceil\log _{2}\left(\left\lceil\frac{n}{2}\right\rceil+1\right)\right\rceil \\
& +5+8 \cdot\left\lceil\log _{2}\left(\left\lceil\frac{n+4}{2}\right\rceil+1\right)\right\rceil \\
& +5+8 \cdot\left\lceil\log _{2}\left(\left\lceil\frac{n+3}{2}\right\rceil+1\right)\right\rceil \\
& +8
\end{aligned}
$$

The width of the output of this operation is $(2 \times n)$ bits. Thus, to reduce the width to fit back in the SDRModAdd, an SDR-to-binary conversion is needed, which is easily 
done as mentioned before using any binary adder (i.e. PPA is the best suite for this in terms of delay). Therefore, the delay of the "division-after-multiplication" becomes:

$$
\begin{aligned}
\text { Delay }_{\text {Division-After-Multiplication }}= & 15+8 \cdot\left\lceil\log _{2}\left(\left\lceil\frac{n}{2}\right\rceil+1\right)\right\rceil \\
& +5+8 \cdot\left\lceil\log _{2}\left(\left\lceil\frac{n+4}{2}\right\rceil+1\right)\right\rceil \\
& +5+8 \cdot\left\lceil\log _{2}\left(\left\lceil\frac{n+3}{2}\right\rceil+1\right)\right\rceil \\
& +8 \\
& +3+2 \cdot\left\lceil\log _{2}(2 n)\right\rceil
\end{aligned}
$$

For this research the size of each channel is $(n=20)$, the delay complexity of "divisionafter-multiplication" is 144 UGD.

For area analysis, the area of "division-after-multiplication", is as follows:

$$
\begin{aligned}
\text { Area }_{\text {Division-After-Multiplication }=} & 1 \times \operatorname{Area}_{S D M u l t}(n) \\
& +1 \times \operatorname{Area}_{S D M u l t}(n+4) \\
& +1 \times \operatorname{Area}_{S D M u l t}(n+3) \\
& +1 \times \operatorname{Area}_{S D S u m}(2 n) \\
& +1 \times \operatorname{Area}_{K S \text { Adder }}(2 n)
\end{aligned}
$$

Where, KSAdder is Kogge-Stone Adder (Kogge \& Stone, 1973) one form of PPA. For the case of $n=20$ the area is $24 k$ UGA.

\subsubsection{Complexity of SDR Base Extension}

As mentioned in section 3.3, the base extension in this research depends on the previous two operations; the SDR modular addition and the SDR modular multiplications. In this research, the base extension is based on the CRT only, due to its characteristic of operating on all operands in parallel. However, extra pre-computation is needed to 
start the process. Recalling the CRT equation is given by:

$$
X=\sum_{1}^{N}\left|x_{i} M_{i}^{-1}\right|_{m_{i}} M_{i}-\alpha \cdot M
$$

and defining:

$$
\xi_{i}=\left|x_{i} M_{i}^{-1}\right|_{m_{i}}
$$

The computation of the correction factor $\alpha$ (as in Equation 4.21):

$$
\alpha=\left.|| M\right|_{m_{x}} ^{-1} \cdot \sum_{1}^{N} \xi_{i} M_{i}-\left.|X|_{m_{x}}\right|_{m_{x}}
$$

is being performed using a redundant modulus (called $m_{x}$ ) (Shenoy \& Kumaresan, 1989) augmented to both moduli sets $\left(M_{\text {set }}\right.$ and $\left.\widetilde{M}_{\text {set }}\right)$. This modulus $m_{x}$ has the special form of $\left(2^{k}\right)$, where $\left(2^{k} \geq N\right)$. $N$ is the number of moduli in the source ${ }^{1}$ moduli set. Since, the modulus $m_{x}$ has the form of $2^{k}$, the modular reduction is performed by truncating the $k$ LSBs. Thus, if the moduli set has 103 moduli and each modulus has the width of 20 bits; the modulus $m_{x}$ can be 10-bit long, which is shorter than any other modulus and its modular computation is much faster and simpler.

As Figure 3.5 shows, the base extension starts with $\left(N_{1} \times N_{2}\right)$ two modular multiplications, where $N_{1}$ and $N_{2}$ are the numbers of moduli in the source moduli set, and the destination moduli set respectively. The first modular multiplication is to compute the terms $\xi_{i}$ and the second modular multiplication is to compute $\left|\xi_{i} M_{i}\right|_{\widetilde{m}_{j}}$. The advantage of the CRT is for the $N_{1} \times N_{2}$ array all the modular multiplications can be performed in parallel at the same time.

The next step is to perform a SDR Modular Addition tree as explained in Figure 3.3 utilizing the design in subsection 4.1 .1 (i.e. SDRModAdd). Therefore, the delay of the base extension is:

$$
\begin{aligned}
\text { Delay }_{\text {SDR Base Exension }=} & 2 \times \text { Delay }_{\text {SDRModMult }} \\
& +1 \times \text { Delay }_{\text {SDR Addition Tree }}
\end{aligned}
$$

\footnotetext{
${ }^{1}$ The source moduli set is the RNS moduli set that number is being transformed from.
} 
where,

$$
\text { Delay }_{\text {SDR Addition Tree }}=\text { Delay }_{S D R M o d A D D} \times \text { Depth of the tree }
$$

and,

$$
\text { Depth }_{\text {SDR Addition Tree }}=\left\lceil\log _{2}(N)\right\rceil
$$

such that, $N$ is the number of operands. To add the term $|-\alpha M|_{\widetilde{m}_{j}}$ an additional step is needed. For example, the delay of the SDR Base Extension for 103 moduli in source set of length 20 bits each to 103 moduli in destination set is:

$$
\begin{aligned}
\text { Delay }_{\text {SDR Base Exension }}(20,103)= & 2 \times \text { Delay }_{\text {SDRModMult }}(21) \\
& +15 \times\left(\left\lceil\log _{2}(103)\right\rceil+1\right) \\
\text { Delay }_{\text {SDR Base Exension }}(20,103)= & 408 \mathrm{UGD}
\end{aligned}
$$

The delay of computing the correction factor $\alpha$ when the redundant modulus $m_{x}$ is of the form of $2^{k}$ is as follows:

$$
\begin{aligned}
\text { Delay }_{\text {Computing } \alpha=} & 3 \times \text { Delay }_{m_{x} \text { Modular Multiplication }} \\
+ & +1 \times \text { Delay }_{m_{x} \text { Modular Adder Tree }} \\
+ & +1 \times \text { Delay }_{\mathrm{KS} \text { Adder }}(k)
\end{aligned}
$$

The last term is added since KS Adder is used to covert the $\alpha$ to binary representation since the value of $\alpha$ is used as an address to the pre-computed values $|-\alpha M|_{\tilde{m}_{j}}$ (as explained in subsection 3.3.1).

In this work the bit-width of the normal moduli is $n=20$ and the number of moduli in the set is 103 moduli. Hence, the width of the redundant modulus $m_{x}$ is $k=8$ bits. The delay of $\alpha$ computation is 178 UGD, which is less than the delay of two SDR modular multiplications is 268 UGD. Thus, the term $|-\alpha M|_{\tilde{m}_{j}}$ can be added at the beginning of the SDR modular addition tree in the base extension. However, the delay 
of SDR Base Extension is:

$$
\begin{aligned}
\text { Delay }_{\text {SDR Base Exension }}(20,103)= & 2 \times \text { Delay }_{\text {SDRModMult }}(21) \\
& +15 \times\left(\left\lceil\log _{2}(103+1)\right\rceil\right) \\
\text { Delay }_{\text {SDR Base Exension }}(20,103)= & 393 \mathrm{UGD}
\end{aligned}
$$

The area of the SDR Base Extension will be as follows:

$$
\begin{aligned}
\text { Area }_{\text {SDR Base Extension }=} & N_{1} \times \operatorname{Area}_{S D R M o d M u l t}(n) \\
+ & N_{2} \times\left(\operatorname{Area}_{\operatorname{SDRModMult}}(n)\right. \\
& \left.+\operatorname{Area}_{\mathrm{SDR} \text { Addition Tree }}\left(n, N_{1}+1\right)\right) \\
& + \text { Area }_{\text {Computing } \alpha \text { the correction factor }}
\end{aligned}
$$

Thus, for $n=20, N_{1}=103$, and $N_{2}=103$, the area is 12.2 MUGA.

\subsection{Complexity Comparison}

Since the goal of this research is to design a RNSMM using general moduli sets (i.e. there is no restriction on the form of the moduli in the sets), designs such as (Kawamura, Komano, Shimizu, \& Yonemura, 2018) is not subject for comparison because they deal with specific moduli sets. Hence, the work to be compared with is (Asif \& Kong, 2017), since it is the most recent work related to this research, and it is also a base for more recent works such as (Asif, Andersson, et al., 2018). Some other works are worth to look at such as (Neto, Tenca, \& Ruggiero, 2014).

Moreover, (Gandino, Lamberti, Paravati, Bajard, \& Montuschi, 2012) did a survey on RNS Montgomery Multiplication analyzing and evaluating different designing approaches. However, since (Asif \& Kong, 2017) is an improvement on (Gandino et al., 2012) the comparison will be held between the proposed design of this research and (Asif \& Kong, 2017). 
As the design and analysis of the proposed design was divided into the three main elements, the comparison will be held in terms of the the three elements, the Modular Addition, the Modular Multiplication and the Base Extension.

\subsubsection{Complexity Comparison of Modular Addition}

In (Asif \& Kong, 2017) the RNS modular addition is done through a binary adder followed by Barrett reduction. The delay of such process is too big compared with the simple fast and fixed complexity delay of the proposed design for the modular addition.

The delay of (Asif \& Kong, 2017)'s modular addition as follows:

$$
\begin{aligned}
\operatorname{Delay}_{(\text {Asif \& Kong, 2017) Modular Additon }=} & \text { Delay }_{\text {Binary Addition }} \\
& + \text { Delay }_{\text {Barret Reduction }}
\end{aligned}
$$

where the fastest binary adders are the PPA such as (Sklansky, 1960), (Kogge \& Stone, 1973) and so forth. The delay of PPA is:

$$
\text { Delay }_{\mathrm{PPA}}=3+2 \cdot\left\lceil\log _{2}(n)\right\rceil
$$

where $n$ is the width of the input. Similarly, the delay of the Barrett reduction is:

$$
\begin{aligned}
\text { Delay }_{\text {Barrett Reduction }=} & 1 \times \text { Delay }_{\text {Binary Multiplication }}(n+4) \\
+ & 1 \times \text { Delay }_{\text {Binary Multiplication }}(n+3) \\
+ & + \text { Delay }_{\text {Binary Addition }}(2 n)
\end{aligned}
$$

Such that the delay of the binary multiplication is:

$$
\begin{aligned}
\text { Delay }_{\text {Binary Multiplication }=} & \text { Delay }_{\text {Multiplier Recoding }}+\text { Delay }_{\text {Partial Products Generating }} \\
& + \text { Delay }_{\text {Wallce Tree }}\left(\frac{n}{2}\right)+\text { Delay }_{\text {Binary Addition }}(2 n) \\
\text { Delay }_{\text {Binary Multiplication }=} & 4+1+4 \cdot\left\lceil\log _{\frac{3}{2}}\left(\frac{n}{2}\right)\right\rceil+3+2 \cdot\left\lceil\log _{2}(2 n)\right\rceil \\
= & 8+4 \cdot\left\lceil\log _{\frac{3}{2}}\left(\frac{n}{2}\right)\right\rceil+2 \cdot\left\lceil\log _{2}(2 n)\right\rceil
\end{aligned}
$$


for $n=20$. Hence, the delay of (Asif \& Kong, 2017) modular addition is 120 UGD. Whereas the proposed design modular addition which requires 17 UGD. Asif and Kong's design is $700 \%$ slower than the proposed design.

Area of (Asif \& Kong, 2017)'s modular addition is computed as follows:

$$
\begin{aligned}
& \text { Area }_{(\text {Asif \& Kong, 2017) Modular Addition }=} \text { Area Binary Addition }(n) \\
&+ \text { Area } \\
& \text { Barrett Reduction }
\end{aligned}
$$

where area of the binary addition using (Kogge \& Stone, 1973) PPA adder is:

$$
\text { Area }_{\mathrm{KS} \text { Adder }}=3 \cdot n \cdot\left\lceil\log _{2}(n)\right\rceil-2 n-3
$$

and the area of the Barrett reduction is:

$$
\begin{aligned}
\text { Area Barrett Reduction }= & \text { Area Binary Multiplication }(n+4) \\
& + \text { Area }_{\text {Binary Mutliplication }}(n+3) \\
& + \text { Area }_{\mathrm{KS} \text { Adder }}
\end{aligned}
$$

and the area of the binary multiplication is:

$$
\begin{aligned}
\text { Area }_{\text {Binary Multiplication }=} & \text { Area Multiplier Recoding }+ \text { AreaPartial Products Generating } \\
& + \text { Area Wallace Tree }\left(\frac{n}{2}, n\right)+\text { Area Binary Addition }(2 n)
\end{aligned}
$$

Thus, for $n=20$, the area of (Asif \& Kong, 2017)'s modular addition is 17.6k UGA. However, the area of the proposed design for $n=20$ is $1.2 \mathrm{k}$ UGA. Hence, (Asif \& Kong, 2017)'s design is $1367 \%$ larger than the proposed design.

Therefore, the proposed design of modular addition outperforms (Asif \& Kong, 2017)'s design of modular addition in both delay and area. In fact, the proposed design is much faster. 


\subsubsection{Complexity Comparison of Modular Multiplication}

Asif and Kong's design of modular multiplication is simply a binary multiplication followed by an improved Barrett reduction (Dhem, 1994). Thus, the delay of their modular multiplier is as follows:

$$
\begin{aligned}
\operatorname{Delay}_{(\text {Asif \& Kong, 2017)'s Modular Multiplier }=} & \text { Delay }_{\text {Binary Multiplier }} \\
& + \text { Delay }_{\text {Barrett Reduction }} \\
\text { Delay }_{(\text {Asif \& Kong, 2017)'s Modular Multiplier }=} & \text { Delay }_{\text {Binary Multiplier }}(n) \\
& + \text { Delay }_{\text {Binary Multiplier }}(n+4) \\
& + \text { Delay }_{\text {Binary Multiplier }}(n+3) \\
& + \text { Delay }_{\text {Binary Adder }}(2 n)
\end{aligned}
$$

where Delay Binary Multiplier was calculated in Equation 4.31, and Delay $_{\text {Binary Adder when }}$ using PPA adder is in Equation 4.28. Therefore, in case of $n=20$ bits, the delay of (Asif \& Kong, 2017)'s modular multiplier is 157 UGD, while the delay of the proposed design's modular multiplier is 144 UGD. This implies that the proposed design is $9 \%$ faster than (Asif \& Kong, 2017)'s design.

The area of (Asif \& Kong, 2017)'s modular multiplier is as follows:

$$
\begin{aligned}
\text { Area }_{(\text {Asif \& Kong, 2017)'s Modular Multiplier }=} & \text { Area Binary Multiplication } \\
& + \text { Area Barrett Reduction }
\end{aligned}
$$

where Equation 4.35 is for estimating the area of the binary multiplier and Equation 4.34 is for estimating the area of the improved Barrett reduction.

The area of (Asif \& Kong, 2017)'s modular multiplier is 24 kUGA for $n=20$ while the area of the proposed design's modular multiplier 28 kUGA. Hence, the proposed design area of modular multiplier is $14 \%$ larger than (Asif \& Kong, 2017). This due to the fact of using the SDR, which utilizes 2 binary bits to represent a SDR bit. 


\subsubsection{Complexity Comparison of Base Extension}

Asif and Kong's design for Base Extension consists of two modular multipliers, a Wallace Tree (Wallace, 1964), a binary adder to compute the final sum the Wallace tree, a binary adder to add the term $|-\alpha M|_{\tilde{m}_{j}}$ and last step is an improved Barrett Reduction circuit. Thus, the delay of (Asif \& Kong, 2017)'s base extension is as follows:

$$
\begin{aligned}
\operatorname{Delay}_{(\text {Asif } \& \text { Kong, 2017)'s Base Extension }=} & 2 \times \text { Delay }_{\text {Modular Multiplier }} \\
& +1 \times \text { Delay }_{\text {Wallace Tree }}\left(N_{1}\right) \\
& +2 \times \text { Delay }_{\text {Binary Addition }}\left(n+\left\lceil\log _{\frac{3}{2}}\left(N_{1}\right)\right\rceil\right) \\
& +1 \times \text { Delay }_{\text {Barrett Reduction }}
\end{aligned}
$$

where $n$ is the width of RNS moduli, and $N_{1}$ is the number of the moduli in the source RNS moduli set. Thus, for $n=20$ and $N_{1}=104^{2}$ the delay is 505 UGD. Even, if they used the same number of moduli in the RNS set as the proposed design (i.e. $N_{1}=103$ ) the delay is still the same (i.e. 505 UGD). Comparing this with the proposed design delay which is 393 UGD, the proposed design is 29\% faster than (Asif \& Kong, 2017)'s design for the base extension.

Area of (Asif \& Kong, 2017)'s base extension consists as follows:

$$
\begin{aligned}
& \text { Area }_{(\text {Asif \& Kong, 2017)'s Base Extension }}=\quad N_{1} \times \text { AreaModular Multiplier } \\
& +N_{2} \times(\text { Area Modular Multiplier }(n) \\
& + \text { Areawallace Tree }\left(n, N_{1}\right) \\
& +\operatorname{Area}_{\text {Binary Addition }}\left(n+\left\lceil\log _{\frac{3}{2}}\left(N_{1}\right)\right\rceil\right) \\
& \left.+\operatorname{Area}_{\text {Binary Addition }}\left(n+\left\lceil\log _{\frac{3}{2}}\left(N_{1}\right)\right\rceil+1\right)\right) \\
& + \text { Area }_{\text {Correction Factor } \alpha \text { Computation }}
\end{aligned}
$$

\footnotetext{
${ }^{2}$ Note in (Asif \& Kong, 2017)'s deign for base extension, since they use approximation methods such as (Kawamura et al., 2000) to compute the value of $\alpha$ they added extra restriction on the available value of the dynamic range of the moduli set (i.e. the allowed number to represent in the moduli set is part of the full dynamic range). Hence, they needed extra moduli to be added to fully cover the range of $[0, M-1]$.
} 
Table 4.3: The values of the parameters used in this research design example.

\begin{tabular}{|c||c|c|}
\hline Value & Proposed & Asif and Kong \\
\hline \hline$n$ & 20 & 20 \\
$N_{1}, N_{2}$ & 103 & 104 \\
$q$ & - & 8 \\
$\delta$ & - & 0.75 \\
\hline
\end{tabular}

Thus, for $n=20, N_{1}=N_{2}=104$, the area of Asif and Kong's base extension design is 13.2 MUGA. Therefore, the proposed design's base extension is $18.5 \%$ larger than (Asif \& Kong, 2017)'s base extension design ${ }^{3}$.

Table 4.4 shows a summary comparison for the example results of the values showed in Table 4.3. The relative performance of the proposed approach is computed using the formula:

$$
\text { Relative Performance }=\frac{\text { Proposed }-(\text { Asif \& Kong, } 2017)}{\text { Proposed }} \times 100 \%
$$

As shown in Table 4.4, the overall improvement in delay is about $28 \%$, while it has a bigger area of $26.4 \%$.

\footnotetext{
${ }^{3}$ The Wallace Tree used for the designs of (Asif \& Kong, 2017) is based on (Asif \& Kong, 2015) which is depending on the design of the (7:3) compressor of (Mehta, Parmar, \& Swartzlander, 1991).
} 


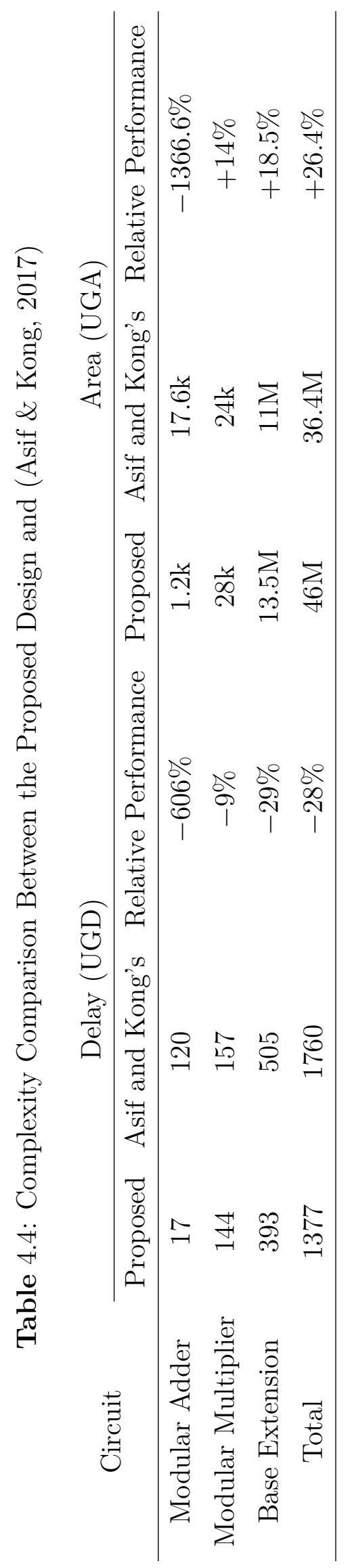




\section{Recommendations and Future Work}

As indicated in Chapter 4 the proposed design outperforms the current designs in terms of delay. One of the most improvements was in Base Extension. The Base Extension -as mentioned in Section 3.3- is a binary-to-RNS embedded in an RNS-tobinary conversion. Thus, a new design for the RNS-to-binary converter for general moduli set is expected to perform faster than recent converters. Moreover, since the conversion from binary to SDR is costless in terms of delay (i.e. it is just an insertion to a zero to the left of each binary bit); the design can run on both binary and SDR.

As mentioned in Subsection 3.2.1, although "division-during-multiplication" needs no extra computation compared to "division-after-multiplication", the former is still slower than the latter. Thus, if it is possible to develop a new design for "divisionduring-multiplication" by skipping some steps and perform modular reduction every other step.

According to the property of fixed-complexity of the SDR addition and modular addition, its applications are countless. With proper treatment, SDR can be used in any other designs such as (Ding \& Li, 2018) and other designs. In addition, this property may be useful in terms of improving this system to prevent power analysis and time analysis (i.e. side-channel attacks).

One of the potential work is to develop a design for point addition and point doubling for ECC. On one hand, ECC key is much smaller than RSA and D-H. On the other hand, the computation of ECC is more than just a modular multiplication. 


\section{References}

Anderson, R., Biham, E., \& Knudsen, L. (1999). Serpent - A New Block Cipher Proposal for AES (Tech. Rep.). NIST.

Antão, S., Bajard, J. C., \& Sousa, L. (2012). RNS-based elliptic curve point multiplication for massive parallel architectures. The Computer Journal, 55(5), 629-647.

Antão, S., \& Sousa, L. (2014). A Flexible Architecture for Modular Arithmetic Hardware Accelerators based on RNS. Journal of Signal Processing Systems, 76(3), 249-259.

Asif, S., Andersson, O., Rodrigues, J., \& Kong, Y. (2018). 65-nm CMOS low-energy RNS modular multiplier for elliptic-curve cryptography. IET Computers $\&$ Digital Techniques, 12(2), 62-67.

Asif, S., Hossain, M. S., Kong, Y., \& Abdul, W. (2018). A Fully RNS based ECC Processor. Integration, 61, 138-149.

Asif, S., \& Kong, Y. (2015). Design of an algorithmic Wallace multiplier using high speed counters. In Proceedings of 2015 Tenth International Conference on Computer Engineering Systems (ICCES) (pp. 133-138). IEEE.

Asif, S., \& Kong, Y. (2017). Highly Parallel Modular Multiplier for Elliptic Curve Cryptography in Residue Number System. Circuits, Systems, and Signal Processing, 36(3), 1027-1051.

Avizienis, A. (1961). Signed-Digit Numbe Representations for Fast Parallel Arithmetic. IRE Transactions on Electronic Computers, EC-10(3), 389-400.

Bajard, J.-C., Didier, L.-S., \& Kornerup, P. (2001). Modular multiplication and base extensions in residue number systems. In Proceedings of 15th IEEE Symposium on Computer Arithmetic. ARITH-15 2001 (pp. 59-65). IEEE Comput. Soc.

Bajard, J. C., Eynard, J., \& Gandino, F. (2013). Fault detection in RNS Montgomery modular multiplication. In Proceedings of 2013 IEEE 21st Symposium on Computer Arithmetic (pp. 119-126).

Bajard, J. C., Kaihara, M., \& Plantard, T. (2009). Selected RNS Bases for Modular Multiplication. In Proceedings of 2009 19th IEEE Symposium on Computer Arithmetic (pp. 25-32). 
Barrett, P. (1987). Implementing the Rivest Shamir and Adleman Public Key Encryption Algorithm on a Standard Digital Signal Processor. In A. M. Odlyzko (Ed.), Proceedings of Advances in Cryptology - CRYPTO' 86 (pp. 311-323). Berlin, Heidelberg: Springer Berlin Heidelberg.

Brent, \& Kung. (1982). A Regular Layout for Parallel Adders. IEEE Transactions on Computers, C-31(3), 260-264.

Burwick, C. D., Coppersmith, D., D'Avignon, E., Gennaro, R., Halevi, S., Jutla, C., ... Zunic, N. (1998). MARS - a candidate cipher for AES (Tech. Rep.). IBM Corporation.

Daemen, J., \& Rijmen, R. V. (1999). AES Proposal: Rijndael (Tech. Rep.). NIST.

Dhem, J. F. (1994). Modified version of the Barret modular multiplication algorithm (Tech. Rep.). Université catholique de Louvain.

Dhem, J. F. (1998). Design of an efficient public-key cryptographic library for RISCbased smart cards (Unpublished doctoral dissertation). Universit Catholique de Louvain.

Diffie, W., Diffie, W., \& Hellman, M. E. (1976). New Directions in Cryptography. IEEE Transactions on Information Theory, 22(6), 644-654.

Dignan, L. (2015). IBM Research builds functional 7nm processor | ZDNet. ZDNet. Retrieved from https://www.zdnet.com/article/ibm-research-builds-functional-7nm -processor/

Ding, J., \& Li, S. (2018). A Modular Multiplier Implemented With Truncated Multiplication. IEEE Transactions on Circuits and Systems II: Express Briefs, 65(11), 1713-1717.

Elgamal, T. (1985). A public key cryptosystem and a signature scheme based on discrete logarithms. IEEE Transactions on Information Theory, 31(4), 469-472.

Gandino, F., Lamberti, F., Paravati, G., Bajard, J.-C., \& Montuschi, P. (2012). An Algorithmic and Architectural Study on Montgomery Exponentiation in RNS. IEEE Transactions on Computers, 61(8), 1071-1083.

Garner, H. L. (1959). The residue number system. In Papers presented at the the March 3-5, 1959, western joint computer conference on XX - IRE-AIEE-ACM 59 (Western) (pp. 146-153). New York, New York, USA: ACM Press.

Gorss, J. (2016). GLOBALFOUNDRIES to Deliver Industry's Leading-Performance Offering of $7 \mathrm{~nm}$ FinFET Technology | GLOBALFOUNDRIES. Global Foun- 
dation, 15. Retrieved from https://www.globalfoundries.com/news-events/ press-releases/globalfoundries-deliver-industrys-leading-performance-offering $-7 \mathrm{~nm}$-finfet

Huang, C. H. (1983). A Fully Parallel Mixed-Radix Conversion Algorithm for Residue Number Applications. IEEE Transactions on Computers, C-32(4), 398-402.

Intel. (2018). Intel Core X-Series Processor Families. Retrieved from https://www.intel.com/content/www/us/en/products/processors/core/6th -gen-x-series-datasheet-vol-1.html

Intel. (2019). Intel Core i־-9700K Processor (12M Cache, up to 4.90 GHz) 186604. Retrieved 2019-03-23, from https://www.intel.com/content/www/us/en/products/ processors/core/i7-processors/i7-9700k.html

Karn, P., Metzger, P., \& Simpson, W. (1995). The ESP Triple DES Transform (Tech. Rep. No. 1851).

Kawamura, S., Koike, M., Sano, F., \& Shimbo, A. (2000). Cox-Rower Architecture for Fast Parallel Montgomery Multiplication. In B. Preneel (Ed.), Proceedings of Advances in Cryptology - EUROCRYPT 2000 (pp. 523-538). Berlin, Heidelberg: Springer Berlin Heidelberg.

Kawamura, S., Komano, Y., Shimizu, H., \& Yonemura, T. (2018). RNS Montgomery reduction algorithms using quadratic residuosity. Journal of Cryptographic Engineering.

King, I. (2018). Samsung Says New 7-Nanometer Chip Production Starting This Year - Bloomberg. Bloomberg, 23. Retrieved from https://www.bloomberg.com/news/ articles/2018-05-22/samsung-says-new-7-nanometer-chip-production-starting -this-year

Koblitz, N. (1987). Elliptic curve cryptosystems. Mathematics of Computation, 48(177), 203-203.

Kogge, P. M., \& Stone, H. S. (1973). A Parallel Algorithm for the Efficient Solution of a General Class of Recurrence Equations. IEEE Transactions on Computers, C-22(8), 786-793.

Mehta, M., Parmar, V., \& Swartzlander, E. (1991). High-speed multiplier design using multi-input counter and compressor circuits. In Proceedings of 10th IEEE Symposium on Computer Arithmetic (pp. 43-50). Grenoble: IEEE Comput. Soc. Press.

Menezes, A. J. A. J., Van Oorschot, P. C., \& Vanstone, S. A. (1997). Number Theory. 
In Handbook of applied cryptography (p. 71). CRC Press.

Miller, V. S. (1986). Use of Elliptic Curves in Cryptography. In H. C. Williams (Ed.), Proceedings of Advances in Cryptology - CRYPTO '85 Proceedings (pp. 417-426). Berlin, Heidelberg: Springer Berlin Heidelberg.

Montgomery, P. L. (1985). Modular multiplication without trial division. Mathematics of Computation, 44(170), 519-521.

Neto, J. C., Tenca, A. F., \& Ruggiero, W. V. (2014). A Parallel and Uniform k-Partition Method for Montgomery Multiplication. IEEE Transactions on Computers, 63(9), 2122-2133.

Orup, H., \& Kornerup, P. (1991). A high-radix hardware algorithm for calculating the exponential M/sup E/ modulo N. In Proceedings of 10th IEEE Symposium on Computer Arithmetic (pp. 51-56). IEEE Comput. Soc. Press.

Rivest, R. L., Robshaw, M. J. B., Sidney, R., \& Yin, Y. L. (1998). The RC6 Block Cipher. In Proceedings of First Advanced Encryption Standard (AES) Conference (p. 16).

Rivest, R. L., Shamir, A., \& Adleman, L. (1978). A method for obtaining digital signatures and public-key cryptosystems. Commun. ACM, 21(2), 120-126.

Schinianakis, D., \& Stouraitis, T. (2011). A RNS Montgomery multiplication architecture. In Proceedings of 2011 IEEE International Symposium of Circuits and Systems (ISCAS) (pp. 1167-1170). IEEE.

Schinianakis, D., \& Stouraitis, T. (2013). An RNS modular multiplication algorithm. In Proceedings of 2013 IEEE 20th International Conference on Electronics, Circuits, and Systems (ICECS) (pp. 958-961). IEEE.

Schneier, B., Kelsey, J., Whiting, D., Wagner, D., \& Chris, H. (1998). Twofish: A 128-Bit Block Cipher (Tech. Rep.). NIST.

Shankland, S. (2018). Apple's A12 Bionic CPU for the new iPhone XS is ahead of the industry moving to $7 \mathrm{~nm}$ chip manufacturing tech. CNET. Retrieved from https:// www.cnet.com/news/iphone-xs-a12-bionic-chip-is-industry-first-7nm-cpu/

Shenoy, A., \& Kumaresan, R. (1989). Fast base extension using a redundant modulus in RNS. IEEE Transactions on Computers, 38(2), 292-297.

Sklansky, J. (1960). Conditional-Sum Addition Logic. IEEE Transactions on Electronic Computers, EC-9(2), 226-231. 
Standard, D. E., \& Others. (1977). Federal information processing standards publication 46. National Bureau of Standards, US Department of Commerce, 4.

Summers, N. (2018). Apple's A12 Bionic is the first 7-nanometer smartphone chip. Engadget. Retrieved from https://www.engadget.com/2018/09/12/apple-a12-bionic -7-nanometer-chip/

Takagi, N. (1992). A radix-4 modular multiplication hardware algorithm for modular exponentiation. IEEE Transactions on Computers, 41(8), 949-956.

Takagi, N., \& Yajima, S. (1992). Modular Multiplication Hardware Algorithms with a Redundant Representation and Their Application to RSA Cryptosystem. IEEE Transactions on Computers, 41(7), 887-891.

Timarchi, S., Saremi, M., Fazlali, M., \& Gaydadjiev, G. (2013). High-speed Binary Signed-Digit RNS adder with posibit and negabit encoding. In Proceedings of 2013 IFIP/IEEE 21st International Conference on Very Large Scale Integration (VLSI-SoC (pp. 58-59).

UV500 2.5"/m.2/mSATA Encrypted SSD - 120GB-1.92TB / Kingston. (2018). Retrieved 2019-03-23, from https://www.kingston.com/us/ssd/business/suv500

Wallace, C. S. (1964). A Suggestion for a Fast Multiplier. IEEE Transactions on Electronic Computers, EC-13(1), 14-17.

Wei, S. (2009). Modular Multipliers Using a Modified Residue Addition Algorithm with Signed-Digit Number Representation. In Proceedings of the International MultiConference of Engineers and Computer Scientists (pp. 494-499). Hong Kong.

Yang, Q., Hu, X., \& Qin, Z. (2015). Secure Systolic Montgomery Modular Multiplier Over Prime Fields Resilient to Fault-Injection Attacks. IEEE Transactions on Very Large Scale Integration (VLSI) Systems, 23(9), 1889-1902.

Yao, G. X., Fan, J., Cheung, R. C., \& Verbauwhede, I. (2014). Novel RNS Parameter Selection for Fast Modular Multiplication. IEEE Transactions on Computers, 63(8), 2099-2105.

Zimmermann, R. (1999). Efficient VLSI implementation of modulo ( $2 \widehat{\mathrm{n}} \pm 1)$ addition and multiplication. In Proceedings of 14th IEEE Symposium on Computer Arithmetic (Cat. No.99CB36336) (pp. 158-167). Adelaide: IEEE Comput. Soc. 


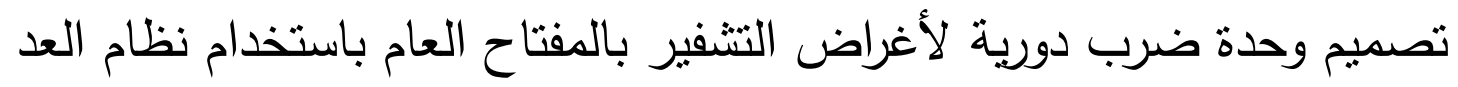
بالبواقي وتمثيل المنازل بالإثارة

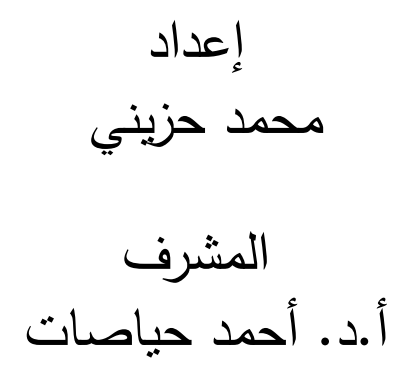

الملخص

إن أنظمة التثفير بالمفتاح العام عرضة لكثير من الطرق التحليلية نتيجة لكونها تعمل بزوج من المفاتيح أحدها

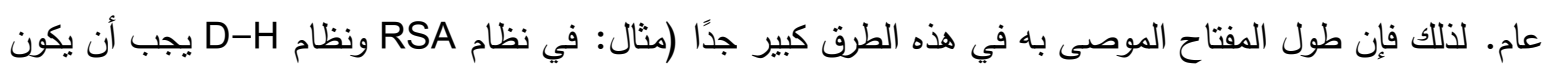
المفتاح على الأقل 2048 خانة ثنائية) وهذا يؤدي إلى أن نكون الحسابات في هذه الأنظمة أبطأ مقارنة بأنظمة التثفير بالمفناح الخاص (AES وعائلة AES). إن العملية الأساسية في هذه الأنظمة هي الضرب وباقي القسمة، وفي هذا البحث نقام تصميمًا حديثًا لهذه العملية، وهو مبني على طريقة مونتجومري في الضرب بالإضافة إلى استخدام نظام العد بالبواقي

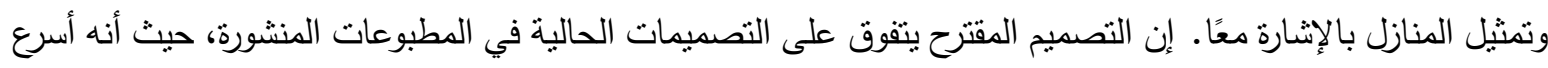
بما لا يقل عن 28\% للمفاتيح بطول 2048 خانة ثنائية. إن هذا التصميم هو الأول من نوعه الذي يجمع بين نظام العد

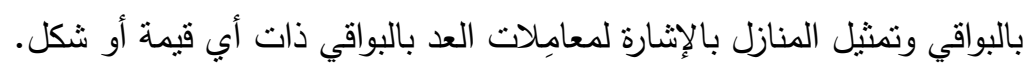

